\title{
Type and extent of trans-disciplinary co-operation to improve food security, health and household environment in low and middle income countries: systematic review
}

Santosh Gaihre ${ }^{1 *}$, Janet Kyle ${ }^{1}$, Sean Semple ${ }^{1}$, Jo Smith², Madhu Subedi ${ }^{3}$ and Debbi Marais ${ }^{4}$

\begin{abstract}
Background: Although linkages have been found between agricultural interventions and nutritional health, and the development of clean fuels and improved solid fuel stoves in reducing household air pollution and adverse health effects, the extent of the potential of combined household interventions to improve health, nutrition and the environment has not been investigated. A systematic review was conducted to identify the extent and type of community-based agricultural and household interventions aimed at improving food security, health and the household environment in low and middle income countries.

Methods: A systematic search of Ovid MEDLINE, PUBMED, EMBASE and SCOPUS databases was performed. Key search words were generated reflecting the "participants, interventions, comparators, outcomes and study design" approach and a comprehensive search strategy was developed following "Preferred Reporting Items for Systematic Reviews and Meta-Analyses" recommendations. Any community-based agricultural and/or household interventions were eligible for inclusion if the focus was to improve at least one of the outcome measures of interest. All relevant study designs employing any of these interventions (alone/in combination) were included if conducted in Low and middle income countries. Review articles, and clinical and occupational studies were excluded.

Results: A total of 123 studies were included and grouped into four intervention domains; agricultural $(n=27)$, air quality $(n=34)$, water quality $(n=32)$, and nutritional $(n=30)$. Most studies were conducted in Asia (39.2 \%) or Africa (34.6\%) with the remaining $26.1 \%$ in Latin America. Very few studies $(n=11)$ combined interventions across more than one domain. The majority of agricultural and nutritional studies were conducted in Africa and Asia, whereas the majority of interventions to improve household air quality were conducted in Latin America.

Conclusions: It is clear that very little trans-disciplinary research has been done with the majority of studies still being discipline specific. It also appears that certain low and middle income countries seem to focus on domain-specific interventions. The review emphasizes the need to develop holistic, cross-domain intervention packages. Further investigation of the data is being conducted to determine the effectiveness of these interventions and whether interdisciplinary interventions provide greater benefit than those that address single health or community problems.
\end{abstract}

Keywords: Agriculture, Food security, Nutrition, Household air pollution, Water quality, Intervention, Health

\footnotetext{
* Correspondence: santosh.gaihre@abdn.ac.uk

${ }^{1}$ Division of Applied Health Sciences, University of Aberdeen, Room 1.019

Polwarth Building, Foresterhill, Aberdeen AB25 2ZD, UK

Full list of author information is available at the end of the article
} 


\section{Background}

Although there has been a significant improvement in global food security, still 805 million people (one in eight people) in low and middle income countries (LMIC) remain chronically undernourished [1]. According to the key findings of the Global Food Security Index 2015 [2], the rate of under nutrition is considerably higher in low and lower middle income countries $(25.4 \%$ and $16.5 \%$ respectively) compared to high income countries (4.9\%). It is also estimated that $29.1 \%$ and $15.5 \%$ of children under the age of five years in lower middle income countries are either stunted or underweight. The prevalence rate is even higher in low income countries where $39.1 \%$ of children under the age of five years are stunted and $22.6 \%$ are underweight [2].

In addition to the health effects of food insecurity leading to poor nutrition, household air pollution from combustion of solid cooking fuels such as firewood, charcoal, etc. is the fourth leading cause of mortality in LMIC [3]. Evidence from epidemiological studies have shown that exposure to household air pollution is associated with acute respiratory tract infection, chronic obstructive pulmonary disease (COPD), cataract and lung cancer [4-6]. Likewise diarrhoea and other common infectious diseases due to poor hygiene and sanitation are also causing significant public health problems in LMIC [3].

It is evident that health is a complex phenomenon determined by multiple risk factors. Complex environmental interactions make it difficult to determine pathways to health in many communities. Food and diet is clearly an important route for exposure to pathogens, but it should not be considered in isolation, since other environmental exposures, such as household air pollution due to burning of biomass for cooking, pesticide exposure from agricultural use and polluted water for drinking, can be equally or more important to health. Food insecurity leading to poor nutrient intake is the main cause of malnutrition, but it is also dependent on other immediate causes, such as the individual's health status [7]. Previous studies have recognised strong linkages between agricultural interventions and nutritional health [8-10] and the development of clean fuels and improved solid fuel stoves in reducing household air pollution and adverse health effects [11]. However, the scale and effectiveness of combined household interventions to improve health, nutrition and the environment has not been investigated. It is unknown whether interventions are inter-disciplinary, crossing domains of health, nutrition, agriculture and/or environment and where these interventions are being conducted. This review determined the extent and types of community-based complex agricultural and household interventions to improve food security, health status and the household environment in LMIC.

\section{Methods}

\section{Search strategy}

A comprehensive search strategy was developed following the recommendations in the PRISMA (Preferred Reporting Items for Systematic Reviews and MetaAnalyses) statement [12]. Key search words were generated reflecting the PICOS (participants, interventions, comparators, outcomes and study design) approach [12]. A database search of Ovid EMBASE was performed using Medical subject heading (MeSH) terms, keywords and truncations covering the potential interventions, outcomes of interest and study design (Additional file 1). The search strategy was developed by combining those search terms using appropriate Boolean operators such as AND/OR/NOT. The search strategy for Ovid MEDLINE, PUBMED and SCOPUS databases were then derived from those search terms and conducted in January 2015. In addition, web and hand searches of bibliographies of identified studies were also performed manually to identify any additional potentially eligible articles.

\section{Study selection and inclusion criteria}

Community-based agricultural and household interventions such as the introduction of biogas, improved cook stoves, home gardening, animal husbandry, livestock farming and nutrition education were eligible to be included in this study if the focus of the intervention was to improve at least one of the outcome measures of interest (Table 1). Human studies employing any of these interventions, alone or in combination, and published after 1990, were included.

The review was open to include any interventional or observational study, such as randomised control trial (RCT), cluster-randomised trial (CRT), cross-sectional study (CSS) and longitudinal studies conducted in LMIC as defined by the World Bank list of economics for 2015. As the main focus of this study was to identify community-based household interventions, clinical and occupational studies were excluded from the review. Similarly, review articles and studies from high income countries were excluded from the review.

All articles identified by electronic searching from the four databases were exported to a web-based bibliography and database manager namely, Refworks. The titles were merged in one database and duplicates removed (Fig. 1). The primary reviewer (SG) screened titles and selected potentially relevant abstracts following predefined inclusion/exclusion criteria. Then four further reviewers (DM, SS, JK and JS) independently examined $10 \%$ of randomly selected titles and abstracts to ensure the accuracy of title and abstract screening process. Disagreements between reviewers were resolved through discussion and checking the full text articles. All articles deemed potentially eligible were retrieved in 
Table 1 Definitions of outcomes of interest measured

\begin{tabular}{|c|c|}
\hline $\begin{array}{l}\text { Outcome } \\
\text { categories }\end{array}$ & Outcomes of interest measured \\
\hline $\begin{array}{l}\text { Food } \\
\text { production }\end{array}$ & $\begin{array}{l}\text { Year round of food production, production of vitamin } \\
\text { A- rich fruits and vegetables, poultry stock and egg } \\
\text { production, fish production, access to goat milk and } \\
\text { other home grown foods }\end{array}$ \\
\hline $\begin{array}{l}\text { Food } \\
\text { consumption }\end{array}$ & $\begin{array}{l}\text { Household food security level/score, Dietary Diversity } \\
\text { Score (DDS), consumption of food/food groups } \\
\text { per day }\end{array}$ \\
\hline Nutrient intake & Micro- and macro-nutrient intake levels \\
\hline Anthropometry & $\begin{array}{l}\text { Prevalence of Stunting [Weight for age Z-score } \\
\text { (WAZ)], Wasting [height for age Z-score (HAZ)], } \\
\text { underweight, child growth, height and weight gain }\end{array}$ \\
\hline $\begin{array}{l}\text { utrient } \\
\text { eficiencies }\end{array}$ & $\begin{array}{l}\text { Vitamin A deficiency level, Incidence/prevalence of } \\
\text { anaemia, serum retinol concentration, serum ferritin } \\
\text { level, haemoglobin, night blindness }\end{array}$ \\
\hline Air quality & $\begin{array}{l}\text { Kitchen/household/personal exposure to carbon } \\
\text { monoxide (CO) and/or concentration of fine particulate } \\
\text { matter of diameter }<2.5 \mu \text { m (PM2.5), kitchen smoke, } \\
\text { suspended particulate matter (PM) concentration, } \\
\text { nitrogen dioxide concentration, ratio of food to fuel }\end{array}$ \\
\hline Health & $\begin{array}{l}\text { Incidence and/or prevalence of: Diarrhoeal disease; } \\
\text { morbidity; respiratory disease symptoms (cough, runny } \\
\text { nose, breathlessness, incidence of chronic obstructive } \\
\text { pulmonary diseases (COPD), pneumonia); eye irritation/ } \\
\text { infection, headache. Changes in: lung function } \\
\text { performance; cognitive performance and attention } \\
\text { levels; quality of life }\end{array}$ \\
\hline $\begin{array}{l}\text { Microbial } \\
\text { Contamination }\end{array}$ & $\begin{array}{l}\text { Thermo tolerant coliforms (TCC) count, level of E.coli } \\
\text { contamination }\end{array}$ \\
\hline $\begin{array}{l}\text { Hygiene and } \\
\text { sanitation }\end{array}$ & $\begin{array}{l}\text { Kitchen and hand hygiene, behaviour and knowledge } \\
\text { of water storage, self-reported compliance }\end{array}$ \\
\hline Education & Perception and knowledge of health and nutrition \\
\hline
\end{tabular}

full text. Reference lists of included studies were also checked to identify other relevant studies.

\section{Data extraction and management}

A standard data extraction form (Additional file 2) was designed considering the Cochrane systematic review data collection checklist [13]. The data collection form was piloted and amended prior to starting the formal data extraction.

Data from all included studies were extracted independently by three reviewers. The extracted data from $10 \%$ of randomly selected articles was then checked independently by a second reviewer to ensure all the correct information was recorded.

\section{Data analysis}

A narrative analysis was conducted based on interventional categorisation. Interventions were categorised according to four domains defined as follows:

- Agricultural interventions: Interventions such as home gardening and animal husbandry that have the explicit goal of improving food productivity, nutritional status, health, dietary diversity and/or food security.

- Air quality interventions: Interventions such as improved cook stove and biogas that have the clear aim of improving household air quality and occupant's health.

- Water quality interventions: Interventions such as water filters (sand and bio sand), solar disinfection technique, water treatment using chlorine tablets alone and/or combination with sanitation health and hygiene education that have the clear aim of improving drinking water quality and health.

- Nutritional interventions: Interventions such as nutrition education, complementary food and nutritional supplements that have the clear aim of improving participants' nutritional status, dietary diversity, and health and food security.

The studies from each interventional category were summarised in tables and narrative text provided to summarise the following aspects:

- country where the study was conducted

- sample size

- setting

- study designs followed

- types of interventions provided

- intervention duration

- outcomes of interest measured

\section{Assessment of methodological quality}

An assessment of the validity of included studies was conducted alongside the data extraction using the Effective Public Health Practice Project (EPHPP) quality assessment tool for quantitative studies [14]. Studies were categorised as strong, moderate or weak based on their quality with regards to component ratings of selection bias, study design, confounders, blinding, data collection method, withdrawals and drop-outs and analysis.

\section{Results}

\section{Identified studies}

The search retrieved 10,847 unique articles (Fig. 1). After removal of 1,638 duplicates the remaining 9,209 articles were screened on the basis of title review. The first stage selection excluded 9,072 articles on the basis of predefined exclusion criteria. Studies were mainly excluded as they were conducted in high income countries, clinical or occupational settings, were not interventional studies or review articles, etc. From these 137 articles were potentially eligible for abstract screening. Finally, 112 articles met the eligibility criteria for the detailed analysis. Of the 25 articles excluded at the abstract screening 
Number of articles identified by search

Embase: 3,368 Medline: 1,134 PubMed: 1,082 Scopus: 5,263

Total: 10,847

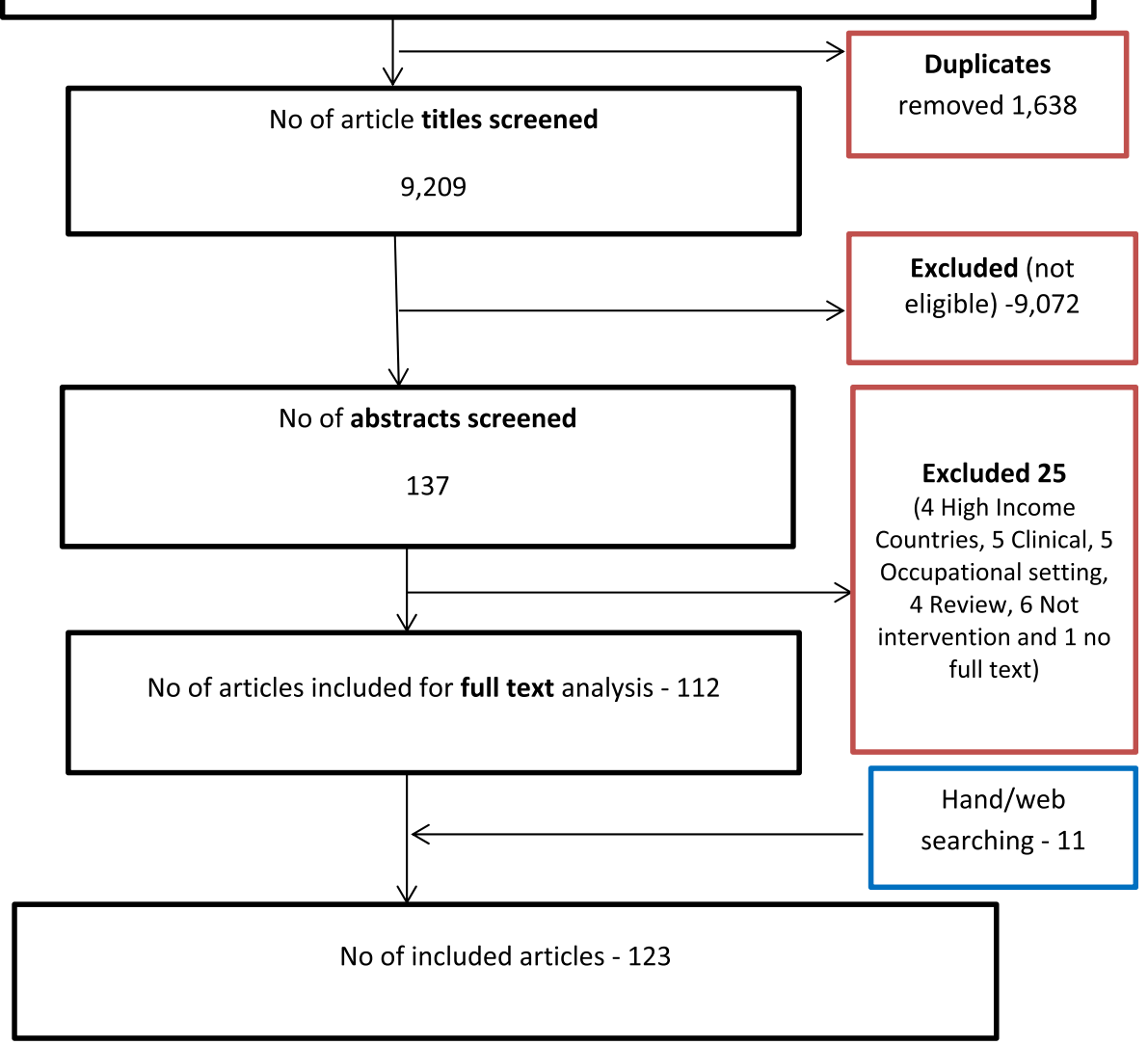

Fig. 1 PRISMA flow diagram

stage four of them were from high income countries, five were in a clinical setting $(\mathrm{Cl})$, five involved occupational settings, four were review articles, six papers were not interventional studies, and the full text of one paper was not available. Eleven additional articles were identified by hand/web searching. Finally, a total of 123 studies were included for the final review.

\section{Study characteristics}

Of the 123 included studies in the review, 27 (21.9\%) were agricultural interventions, 34 (27.6\%) were air quality interventions, 32 (26\%) were water quality interventions and 30 (24.3\%) were nutritional interventions (Fig. 2).

\section{Characteristics of agricultural interventions $(n=27)$}

Of the 27 studies (Table 2) reporting agricultural interventions, 14 projects promoted and supported home gardening and household food production or the improvement of the existing garden with micronutrientrich fruit and vegetables. Six projects promoted animal husbandry, such as pig and poultry breeding, goat farming, fisheries and dairy production. Five studies observed the effectiveness of combined home gardening and nutrition education intervention. One promoted home gardening with animal husbandry and another, a combination of home gardening, animal husbandry and nutrition education.

Most of the studies were either cross sectional $(n=10)$ or intervention studies $(n=10)$ with one RCT [15]. There was a wide variation of sample sizes, ranging from 58 households [16] to $>10,000$ participants [15]. Similarly, duration of the studies varied; from a dairy intensifying intervention in Kenya for two months [17] to a home gardening study in India for 96 months [18]. Fourteen of these studies were conducted in Asia and the other 13 in Africa. The first home gardening study was conducted in Bangladesh in 1996 [19]. Most of these 


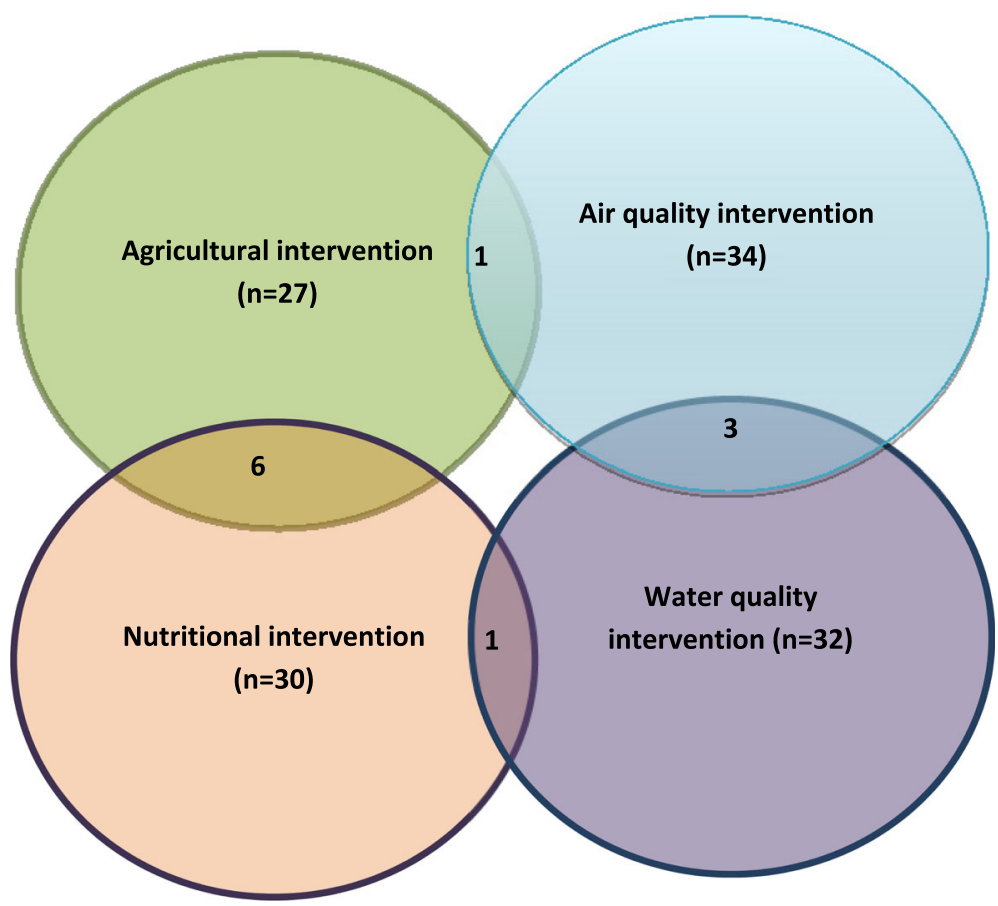

Fig. 2 Overlapping intervention domains

studies $(n=22)$ were conducted in a household setting and only a few in community settings.

Nineteen of these studies examined the effect of intervention on dietary diversity and improvement in food consumption, seven on food production, seven on nutrient intake, seven on nutritional deficiencies, seven on anthropometry, three on education, two on health and two on food security.

\section{Characteristics of air quality interventions $(n=34)$}

Of the 34 air quality studies (Table 3 ), four projects introduced biogas [13-20] as an alternative means of cooking fuel, 17 projects promoted improved cook stoves and 11 studies examined the effectiveness of improved stoves with chimney to improve the household air quality. One project evaluated the impact of improved cook stoves with solar water disinfection and hand hygiene [21], and another looked at an improved cook stove intervention with biogas fuel and solar heaters [20].

Most of the studies provided data either on pre and post or between group comparisons with nine randomised control trial. The sample sizes of the studies ranged from 11 [22] to 4,000 households [23]. The duration of the study also varied considerably; a Peru cook stove project lasted for 3 weeks [24], while one vented stove project in the highlands of Guatemala collected data for 48 months [23]. The majority of the studies $(n=18)$ were conducted in South America, nine were in Asia, with the other seven in African countries. The first cook-stove intervention study was conducted in Nepal in 1990 [25]. All of these studies were conducted in household settings.

Almost all of the studies (28 out of 34) examined the improvement in household air quality parameters such as particulate matter and carbon monoxide concentrations. Twenty studies assessed the impact of the intervention on participants' health outcomes such as incidence of pneumonia, acute respiratory infections (ARI), conjunctivitis and lung function, and three examined the impact on food production.

\section{Characteristics of water quality interventions $(n=32)$}

Of the 32 water quality intervention studies (Table 4), 12 were water filter interventions; nine were chlorine tablets/solutions interventions, seven were Solar disinfection; two were hand water pumps along with hygiene education and latrine construction interventions [26]; one was a health, hand hygiene, water quality and sanitation educational intervention [27]; one involved disinfection tablets along with sanitation and hygiene education [28]; one was a water disinfection stove [29] and one a filter along with improved cook stove [30].

Most of the studies were RCT $(n=25)$ or intervention studies $(n=4)$. The sample sizes of the studies ranged from 2 [29] to 2,193 households [31] and the interventions were delivered over periods of 2 [29] to 15 [32] months. Nine studies were conducted in South America, 
Table 2 Characteristics of agricultural intervention studies

\begin{tabular}{|c|c|c|c|c|c|c|}
\hline $\begin{array}{l}\text { Study (Author and } \\
\text { publication year) }\end{array}$ & Country & $\begin{array}{l}\text { Participants } \\
\text { (sample size, age, setting) }\end{array}$ & Study design & $\begin{array}{l}\text { Intervention details } \\
(I=\text { Intervention and } C=\text { Control })\end{array}$ & $\begin{array}{l}\text { Duration of intervention } \\
\text { (months) }\end{array}$ & Outcome measured \\
\hline $\begin{array}{l}\text { Ayele } Z \text { and } \\
\text { Peacock C; } 2003\end{array}$ & Ethiopia & 210 households & CSS (Pre and post) & I: Animal husbandry: goat farming & NR & $\begin{array}{l}\text { Food consumption, nutrient } \\
\text { deficiencies }\end{array}$ \\
\hline $\begin{array}{l}\text { Belachew T et al. } \\
2013\end{array}$ & Ethiopia & $\begin{array}{l}2100 \text { adolescents, } 13-17 \text { years, } \\
\text { household }\end{array}$ & $\begin{array}{l}5 \text { year } \\
\text { Longitudinal } \\
\text { study }\end{array}$ & I: Food production & NR & Food consumption \\
\hline $\begin{array}{l}\text { Bezner KR, et al. } \\
2010\end{array}$ & Malawi & $\begin{array}{l}3838 \text { children }<3 \text { years, } \\
\text { household }\end{array}$ & $\begin{array}{l}\text { Prospective quasi- } \\
\text { experimental } \\
\text { study }\end{array}$ & $\begin{array}{l}\text { I: Intercropping legumes and nutrition education } \\
\text { C: Usual practice }\end{array}$ & 72 & Anthropometry \\
\hline $\begin{array}{l}\text { Bloem MW et al. } \\
1996\end{array}$ & Bangladesh & $\begin{array}{l}7341 \text { participants, all aged, } \\
\text { household }\end{array}$ & Intervention study & I: Home gardening & NR & Food production \\
\hline $\begin{array}{l}\text { Bushamuka VN, } \\
\text { et al. } 2005\end{array}$ & Bangladesh & 2,160 households & Intervention study & $\begin{array}{l}\text { I: Home gardening } \\
\text { C: Usual practice }\end{array}$ & NR & $\begin{array}{l}\text { Food production, food } \\
\text { consumption }\end{array}$ \\
\hline $\begin{array}{l}\text { Cabalda AB, et al. } \\
2011\end{array}$ & Philippines & $\begin{array}{l}200 \text { households, participants } \\
\text { aged } 2-5 \text { years }\end{array}$ & $\begin{array}{l}\text { CSS ( } 2 \text { group } \\
\text { comparison) }\end{array}$ & $\begin{array}{l}\text { I: Home gardening }(n=105) \\
\text { C: Without home garden }(n=95)\end{array}$ & NR & Food consumption \\
\hline $\begin{array}{l}\text { Faber M, et al. } \\
\text { 2002, }\end{array}$ & $\begin{array}{l}\text { South } \\
\text { Africa }\end{array}$ & $\begin{array}{l}208 \text { participants, aged } 2-5 \\
\text { years, community }\end{array}$ & CSS (Pre and post) & $\begin{array}{l}\text { I: Home gardening and nutrition education } \\
(n=108) \\
\text { C: Usual practice }(n=100)\end{array}$ & 20 & $\begin{array}{l}\text { Food consumption, nutrient } \\
\text { intake, nutrient deficiencies }\end{array}$ \\
\hline $\begin{array}{l}\text { Gibson RS et al. } \\
2003\end{array}$ & Malawi & $\begin{array}{l}281 \text { households, aged } 30-90 \\
\text { months }\end{array}$ & Intervention study & $\begin{array}{l}\text { I: Multiple: Animal husbandry and home gardening } \\
(n=200) \\
\text { C: Usual practice }(n=81)\end{array}$ & 12 & $\begin{array}{l}\text { Food consumption, } \\
\text { anthropometry, education, } \\
\text { nutrient deficiencies, health }\end{array}$ \\
\hline Haseen F, 2007 & Bangladesh & $\begin{array}{l}370 \text { households, all age } \\
\text { participants }\end{array}$ & CSS (Pre and post) & $\begin{array}{l}\text { I: Home based food production, increased } \\
\text { purchasing capacity to improve food intake and } \\
\text { nutritional status }(n=180) \\
\text { C: Usual practice }(n=193)\end{array}$ & 24 & $\begin{array}{l}\text { Food consumption, nutrient } \\
\text { intake }\end{array}$ \\
\hline $\begin{array}{l}\text { Hoorweg J, et al. } \\
2000\end{array}$ & Kenya & $\begin{array}{l}144 \text { households, participants } \\
\text { aged between 6-59 months }\end{array}$ & Intervention study & $\begin{array}{l}\text { I: Dairy farming }(n=30) \text { and dairy customers } \\
(n=24) \\
\text { C: Usual practice }(n=90)\end{array}$ & NR & $\begin{array}{l}\text { Food consumption, } \\
\text { anthropometry, income }\end{array}$ \\
\hline Hop LT; 2003 & Vietnam & NR & $\begin{array}{l}\text { Longitudinal } \\
\text { survey (LS) (pre } \\
\text { and post) }\end{array}$ & I: Programs to improve pig and poultry breeding & NR & $\begin{array}{l}\text { Food consumption, nutrient } \\
\text { deficiencies }\end{array}$ \\
\hline Hotz C, et al. 2012 & Uganda & $\begin{array}{l}>10,000 \text { households, } \\
\text { community }\end{array}$ & $\begin{array}{l}\text { Randomised } \\
\text { control trial }(\mathrm{RCT})\end{array}$ & $\begin{array}{l}\text { 11: B-carotene-rich orange sweet potato (OSP) } \\
\text { vines with training ( } n=293 \text { children, } 212 \text { women) } \\
\text { I2: Education on female and child health and } \\
\text { promotion of OSP ( } n=179 \text { children, } 130 \text { women) } \\
\text { C: Usual practice ( } n=280 \text { children, } 213 \text { women) }\end{array}$ & 12 and 24 & $\begin{array}{l}\text { Nutrient intake, nutrient } \\
\text { deficiencies }\end{array}$ \\
\hline $\begin{array}{l}\text { Jones KM, et al. } \\
2005\end{array}$ & Nepal & 819 households, community & Intervention study & $\begin{array}{l}\text { I: Home gardening and nutrition education } \\
(n=430) \\
\text { C: Usual practice }(n=389)\end{array}$ & 36 & Food consumption, education \\
\hline \multirow[t]{2}{*}{$\begin{array}{l}\text { Kalavathi S, et al. } \\
2010\end{array}$} & India & 150 household & $\begin{array}{l}\text { Intervention study } \\
\text { (pre and post) }\end{array}$ & $\begin{array}{l}\text { I: Package intervention of nutrition gardening, } \\
\text { livestock rearing and nutrition education }\end{array}$ & 36 & $\begin{array}{l}\text { Food production, food } \\
\text { consumption and nutrient } \\
\text { intake }\end{array}$ \\
\hline & Malawi & & Intervention study & & 72 & Anthropometry \\
\hline
\end{tabular}


Table 2 Characteristics of agricultural intervention studies (Continued)

\begin{tabular}{|c|c|c|c|c|c|c|}
\hline $\begin{array}{l}\text { Kerr RB, et al. } \\
2010\end{array}$ & & $\begin{array}{l}3838 \text { participants, aged }< \\
3 \text { years, households }\end{array}$ & & $\begin{array}{l}\text { I: Home gardening and nutrition education } \\
(n=1724) \\
\text { C: Usual practice }\end{array}$ & & \\
\hline $\begin{array}{l}\text { Kidala D, et al. } \\
2000\end{array}$ & Tanzania & 2250 household & $\begin{array}{l}\text { Quasi- } \\
\text { experimental }(2 \\
\text { groups } \\
\text { comparison) }\end{array}$ & $\begin{array}{l}\text { I: Horticultural and nutrition education } \\
\text { ( } n=125 \text { households) } \\
\text { C: Usual practice ( } n=125 \text { households) }\end{array}$ & 60 & $\begin{array}{l}\text { Nutritional knowledge, nutrient } \\
\text { intake, nutrient deficiencies }\end{array}$ \\
\hline $\begin{array}{l}\text { Low JW, et al. } \\
2007\end{array}$ & Mozambiqu & $\begin{array}{l}741 \text { children aged } 13 \text { months, } \\
\text { household }\end{array}$ & $\begin{array}{l}\text { Quasi- } \\
\text { experimental ( } 2 \\
\text { groups } \\
\text { comparison) }\end{array}$ & $\begin{array}{l}\text { I: Production of Orange-fleshed sweet potato } \\
\text { (OFSP) and nutritional knowledge }(n=498) \\
\text { C: Usual practice }(n=243)\end{array}$ & 24 & $\begin{array}{l}\text { Nutrient intake, nutrient } \\
\text { deficiencies }\end{array}$ \\
\hline $\begin{array}{l}\text { Miura S, et al. } \\
2003\end{array}$ & Philippines & 152 women, household & $\begin{array}{l}\text { CSS (pre and } \\
\text { post) }\end{array}$ & I: Home gardening & NR & Food consumption \\
\hline $\begin{array}{l}\text { Murshed-e-Jahan } \\
\text { K, et al. } 2010\end{array}$ & Bangladesh & NR & Intervention study & $\begin{array}{l}\text { I: Training support to farmers on aquaculture } \\
\text { C: Usual practice }\end{array}$ & NR & $\begin{array}{l}\text { Food production, food } \\
\text { consumption }\end{array}$ \\
\hline $\begin{array}{l}\text { Nielsen H, et al. } \\
2003\end{array}$ & Bangladesh & $\begin{array}{l}70 \text { households, women of } \\
\text { reproductive age and 5-12 } \\
\text { years old girls }\end{array}$ & Intervention study & $\begin{array}{l}\text { I: Poultry production }(n=35) \\
\text { C: Usual practice }(n=35)\end{array}$ & 12 & $\begin{array}{l}\text { Food production, food } \\
\text { consumption }\end{array}$ \\
\hline $\begin{array}{l}\text { Olney DK, et al. } \\
2009\end{array}$ & Cambodia & 500 households & CSS (Pre and post) & $\begin{array}{l}\text { I: Home gardening }(n=300) \\
\text { C: Usual practice }(n=200)\end{array}$ & NR & $\begin{array}{l}\text { Food consumption, } \\
\text { anthropometry, health }\end{array}$ \\
\hline $\begin{array}{l}\text { Schipani S, et al. } \\
2002\end{array}$ & Thailand & 60 children, household & Intervention study & $\begin{array}{l}\text { I: Mixed home gardening }(n=30) \\
\text { C: Non gardening }(n=30)\end{array}$ & NR & $\begin{array}{l}\text { Food consumption, } \\
\text { anthropometry }\end{array}$ \\
\hline $\begin{array}{l}\text { Schmid M et al. } \\
2007\end{array}$ & India & $\begin{array}{l}220 \text { participants, Child: } 6 \text { to } \\
39 \text { months and mother > } \\
15 \text { years, community }\end{array}$ & $\begin{array}{l}\text { CSS (pre and } \\
\text { post) }\end{array}$ & $\begin{array}{l}\text { I: Home gardening }(n=124) \\
\text { C: Without home garden (96) }\end{array}$ & 96 & Nutrient intake \\
\hline Sha KK et al. 200, & Bangladesh & $\begin{array}{l}1343 \text { participants aged } \\
<24 \text { months, households }\end{array}$ & $\begin{array}{l}\text { Longitudinal } \\
\text { study }\end{array}$ & $\begin{array}{l}\text { I: Household production and availability of rice and } \\
\text { other fresh foods e.g. Vegetables, fish, meat }\end{array}$ & NR & $\begin{array}{l}\text { Food consumption, } \\
\text { anthropometry }\end{array}$ \\
\hline $\begin{array}{l}\text { Smitasiri et al. } \\
1999\end{array}$ & Thailand & 15 communities, all age & $\begin{array}{l}\text { CSS (pre and } \\
\text { post) }\end{array}$ & $\begin{array}{l}\text { I: Home gardening (seed grant) and nutrition and } \\
\text { health messages ( } 271) \\
\text { C: without home gardening (247) }\end{array}$ & & $\begin{array}{l}\text { Food consumption, nutrient } \\
\text { intake }\end{array}$ \\
\hline $\begin{array}{l}\text { Wyatt AJ, et al. } \\
2013\end{array}$ & Kenya & 92 households & $\begin{array}{l}\text { CSS ( } 3 \text { group } \\
\text { comparison) }\end{array}$ & $\begin{array}{l}\text { Dairy intensification } \\
\text { 11: Milk production }>6 \text { I per day }(n=31) \\
\text { I2: Milk production }<6 \text { I per day }(n=31) \\
\text { C: No milk production }(n=30)\end{array}$ & 2 & Food consumption \\
\hline $\begin{array}{l}\text { Yakubu A, et al. } \\
2014\end{array}$ & Nigeria & 58 households, community & $\begin{array}{l}\text { CSS (pre and } \\
\text { post) }\end{array}$ & I: Cockerel exchange programme & NR & Food production \\
\hline
\end{tabular}

$R C T$ randomised control trial, CSS cross sectional study, NR not reported 
Table 3 Characteristics of air quality intervention studies

\begin{tabular}{|c|c|c|c|c|c|c|}
\hline $\begin{array}{l}\text { Study (Author and } \\
\text { publication year) }\end{array}$ & Country & $\begin{array}{l}\text { Participants } \\
\text { (sample size, age, setting) }\end{array}$ & Study design & $\begin{array}{l}\text { Intervention details } \\
(\mathrm{l}=\text { Intervention and } \mathrm{C}=\text { Control })\end{array}$ & $\begin{array}{l}\text { Duration of intervention } \\
\text { (months) }\end{array}$ & Outcome measured \\
\hline $\begin{array}{l}\text { Alexander D, } \\
\text { et al. } 2013\end{array}$ & Bolivia & 31 household & $\begin{array}{l}\text { Intervention study } \\
\text { (pre and post) }\end{array}$ & $\begin{array}{l}\text { I: Improved cook stoves with chimney } \\
\text { (Yanalo Cookstoves) }\end{array}$ & 12 & Air quality, health \\
\hline $\begin{array}{l}\text { Burwen J and } \\
\text { Levine Dl; } 2012\end{array}$ & Ghana & 768 household & RCT & $\begin{array}{l}\text { I: Improved cook stoves with chimney }(n=402) \\
\text { C: Traditional biomass stoves (usual practice) } \\
(n=366)\end{array}$ & 2 & $\begin{array}{l}\text { Air quality, health, } \\
\text { stove usages }\end{array}$ \\
\hline $\begin{array}{l}\text { Chengappa C, } \\
\text { et al. } 2007\end{array}$ & India & 60 , household & $\begin{array}{l}\text { Paired, before and } \\
\text { after study }\end{array}$ & I: improved cook stoves (Sukhad) & 12 & Air quality \\
\hline $\begin{array}{l}\text { Clark LM, et al. } \\
2009\end{array}$ & Honduras & $\begin{array}{l}79 \text { participants, mean age } 43.2 \text { years, } \\
\text { household, }\end{array}$ & CSS (pre and post) & $\begin{array}{l}\text { I: Improved cook stoves with chimney }(n=38) \\
\text { C: Traditional cook stoves }(n=41)\end{array}$ & 3 & Air quality, health \\
\hline $\begin{array}{l}\text { Chowdhury Z } \\
\text { et al. } 2012\end{array}$ & China & 30 household & CSS (pre and post) & $\begin{array}{l}\text { I: Improved stoves along with biogas burners } \\
\text { and solar heaters }\end{array}$ & 2 & Air quality \\
\hline $\begin{array}{l}\text { Commodore AA, } \\
\text { et al. } 2013\end{array}$ & Peru & 84 participants household & $\begin{array}{l}\text { Community-RCT (C- } \\
\text { RCT) }\end{array}$ & $\begin{array}{l}\text { I: Improved cook stoves (OPTIMA) }(n=39) \\
\text { C: Traditional biomass stove, NGO Stoves, } \\
\text { self-improved stove }(n=45)\end{array}$ & 3 & Air quality, health \\
\hline $\begin{array}{l}\text { Cynthia AA, et al. } \\
2008\end{array}$ & Mexico & 34 households, & Randomised trial & I: Improved cook stoves $(n=60)$ & 1 & Air quality \\
\hline Diaz E, et al. 2008 & Guatemala & $\begin{array}{l}180 \text { women, mean age } 27.8 \text { years, } \\
\text { household }\end{array}$ & $\mathrm{RCT}$ & $\begin{array}{l}\text { I: Improved cook stoves with chimney (Plancha) } \\
(n=89) \\
\text { C: Traditional biomass stove (usual practice) }(n=91)\end{array}$ & 26 & Air quality, health \\
\hline Diaz E, et al. 2007 & Guatemala & 504 women, 27.7 years, household & $\mathrm{RCT}$ & $\begin{array}{l}\text { I: Improved cook stoves with chimney (Plancha) } \\
(n=259) \\
\text { C: Traditional biomass stove (usual practice) } \\
(n=245)\end{array}$ & 18 & Air quality, health \\
\hline $\begin{array}{l}\text { Dohoo C, et al. } \\
2012\end{array}$ & Kenya & 62 women, household & $\begin{array}{l}\text { CSS (comparison } \\
\text { between } 2 \text { groups) }\end{array}$ & $\begin{array}{l}\text { I: Biogas }(n=31) \\
\text { C: Traditional biomass stove }(n=31\end{array}$ & 2 & Health \\
\hline $\begin{array}{l}\text { Ezzati M, et al. } \\
2000\end{array}$ & Kenya & 38 households & Intervention study & I: Improved cook stoves & 1 & Air quality \\
\hline $\begin{array}{l}\text { Fitzgerald C, et al. } \\
2012\end{array}$ & Peru & $\begin{array}{l}57 \text { participants, mean age } 33 \text { years, } \\
\text { household }\end{array}$ & $\begin{array}{l}\text { Intervention study } \\
\text { (pre and post) }\end{array}$ & $\begin{array}{l}\text { I: Improved cook stoves ( } n=26 \text { for PM2.5 and } 25 \\
\text { for CO) }\end{array}$ & 5 & Air quality \\
\hline $\begin{array}{l}\text { Garfi M, et al. } \\
2012\end{array}$ & Peru & 12 households & Intervention study & I: Low-cost tabular biogas digester & NR & $\begin{array}{l}\text { Food production, air } \\
\text { quality }\end{array}$ \\
\hline $\begin{array}{l}\text { Harris SA, et al. } \\
2010\end{array}$ & Guatemala & 4000, household & $\begin{array}{l}\text { Intervention study } \\
\text { (pre and post) }\end{array}$ & $\begin{array}{l}\text { I: Improved cook stoves } \\
\text { C: Traditional biomass stove (usual practice) }\end{array}$ & 48 & Health \\
\hline $\begin{array}{l}\text { Hartinger SM, } \\
\text { et al. } 2012\end{array}$ & Peru & 115 households, household, & $\begin{array}{l}\text { Intervention study } \\
\text { (pre and post) }\end{array}$ & $\begin{array}{l}\text { I: Multiple intervention; improved cook stoves, solar } \\
\text { water disinfection and hand hygiene }\end{array}$ & 5 & $\begin{array}{l}\text { Air quality, hygiene and } \\
\text { sanitation, health }\end{array}$ \\
\hline $\begin{array}{l}\text { Jary HR, et al. } \\
2014\end{array}$ & Malawi & $\begin{array}{l}51 \text { Women, mean age } 38.1 \text { years, } \\
\text { households }\end{array}$ & RCT & $\begin{array}{l}\text { I: Improved cook stoves ( } n=25) \\
\text { C: Traditional biomass stove (usual practice) } \\
(n=26)\end{array}$ & 2 & Air quality, health \\
\hline $\begin{array}{l}\text { Katwal H, Bohara } \\
\text { AK; } 2009\end{array}$ & Nepal & 461 households & Intervention study & I: Biogas digester & NR & $\begin{array}{l}\text { Air quality, health, Food } \\
\text { production }\end{array}$ \\
\hline
\end{tabular}


Table 3 Characteristics of air quality intervention studies (Continued)

\begin{tabular}{|c|c|c|c|c|c|c|}
\hline $\begin{array}{l}\text { Khushk WA, et al. } \\
2005\end{array}$ & Pakistan & $\begin{array}{l}159 \text { women, mean age } 43.27 \text { (I) and } \\
36.18 \text { (C) years, household }\end{array}$ & $\begin{array}{l}\text { CSS (comparison } \\
\text { between } 2 \text { groups) }\end{array}$ & $\begin{array}{l}\text { I: Improved cook stoves ( } n=45) \\
\text { C: Traditional biomass stove (usual practice) }(n= \\
\text { 114) }\end{array}$ & 2 & Air quality, health \\
\hline Li Z, et al. 2011 & Peru & $\begin{array}{l}57 \text { households, participants } \\
\text { aged } 18-45 \text { years, household }\end{array}$ & $\begin{array}{l}\text { Intervention study } \\
\text { (pre and post) }\end{array}$ & I: Improved cooking stove with chimney & 3 weeks & Air quality \\
\hline $\begin{array}{l}\text { McCracken JP, } \\
\text { et al. } 1998\end{array}$ & Guatemala & 11, household & $\begin{array}{l}\text { CSS (comparison } \\
\text { between } 2 \text { groups) }\end{array}$ & $\begin{array}{l}\text { I: Improved cook stoves ( } n=6) \\
\text { C: Traditional biomass stove (usual practice) } \\
(n=5)\end{array}$ & NR & Air quality \\
\hline $\begin{array}{l}\text { McCracken JP, } \\
\text { et al. } 2011\end{array}$ & Guatemala & 534 Households & RCT & $\begin{array}{l}\text { I: Improved stove with Chimney }(n=49) \\
\text { C: Traditional open fire stoves }(n=70)\end{array}$ & 16 & Air quality, health \\
\hline $\begin{array}{l}\text { Mukhopadhyay R, } \\
\text { et al. } 2012\end{array}$ & India & $\begin{array}{l}32 \text { women, mean age } \\
32 \text { years, household }\end{array}$ & CSS (pre and post) & $\begin{array}{l}\text { I: Improved cook stoves } \\
\text { C: Traditional open fire biomass stove } \\
\text { (usual practice) }\end{array}$ & 3 & $\begin{array}{l}\text { Air quality, acceptability } \\
\text { and usage }\end{array}$ \\
\hline $\begin{array}{l}\text { Ochieng CA, } \\
\text { et al. } 2012\end{array}$ & Kenya & 104 Women, household & $\begin{array}{l}\text { CSS (comparison } \\
\text { between } 2 \text { groups) }\end{array}$ & $\begin{array}{l}\text { I: Improved stoves without chimney }(n=49) \\
\text { C: Traditional stoves }(n=45)\end{array}$ & 6 & Air quality \\
\hline $\begin{array}{l}\text { Oluwole O, et al. } \\
2013\end{array}$ & Nigeria & $\begin{array}{l}59 \text { participants, mothers } 43 \\
\text { years and children } 13 \text { years, household }\end{array}$ & CSS (pre and post) & I: Improved stoves & 12 & Air quality, health \\
\hline $\begin{array}{l}\text { Pandey MR, et al. } \\
1990\end{array}$ & Nepal & 20 households & Intervention study & I: Improved cook stoves $(n=20)$ & 5 & Air quality \\
\hline $\begin{array}{l}\text { Riojas-Rodriguez, } \\
\text { et al. } 2011\end{array}$ & Mexico & $\begin{array}{l}47 \text { women, mean age } 28 \text { years, } \\
\text { household }\end{array}$ & $\mathrm{RCT}$ & $\begin{array}{l}\text { I: Improved cook stoves fitted with chimney } \\
\text { (Patsari stoves) }(n=30) \\
\text { C: Traditional stoves }(n=17)\end{array}$ & 12 & Air quality \\
\hline $\begin{array}{l}\text { Romieu l, et al. } \\
2009\end{array}$ & Mexico & $\begin{array}{l}528 \text { women, mean age } 26.3 \text { (I) and } \\
25.5 \text { (C) years, household }\end{array}$ & $\mathrm{RCT}$ & $\begin{array}{l}\text { I: Improved cook stoves fitted with chimney } \\
\text { (Patsari stoves) }(n=273) \\
\text { C: Traditional stoves }(n=255)\end{array}$ & 10 & Health \\
\hline $\begin{array}{l}\text { Schilmann A, } \\
\text { et al. } 2014\end{array}$ & Mexico & 559 children $<4$ years, household & $\mathrm{RCT}$ & $\begin{array}{l}\text { I: Improved cook stoves fitted with chimney } \\
\text { (Patsari stoves) }(n=287) \\
\text { C: Traditional stoves }(n=272)\end{array}$ & 10 & Health \\
\hline $\begin{array}{l}\text { Singh A, et al. } \\
2012\end{array}$ & Nepal & 47 households, all aged participants & CSS (pre and post) & I: Improved mud stoves & 12 & Air quality, health \\
\hline $\begin{array}{l}\text { Singh S, et al. } \\
2014\end{array}$ & India & 75 household & $\begin{array}{l}\text { CSS (comparison } \\
\text { between } 2 \text { groups) }\end{array}$ & $\begin{array}{l}\text { I: Improved stoves } \\
\text { C: Traditional stoves }\end{array}$ & 2 & Air quality \\
\hline $\begin{array}{l}\text { Smith KR, et al. } \\
2011\end{array}$ & Guatemala & $\begin{array}{l}534 \text { households, participants aged } \\
<4 \text { months at baseline }\end{array}$ & RCT & $\begin{array}{l}\text { I: Improved wood stove with chimney }(n=265) \\
\text { C: Open wood fires }(n=253)\end{array}$ & 14 & Health \\
\hline $\begin{array}{l}\text { Wafula EM, et al. } \\
2000\end{array}$ & Kenya & $\begin{array}{l}400 \text { households, women aged 15-60 } \\
\text { years and children }<5 \text { years }\end{array}$ & $\begin{array}{l}\text { Intervention study } \\
\text { (pre and post) }\end{array}$ & $\begin{array}{l}\text { I: Improved cook stoves }(n=200) \\
\text { C: Traditional three-stone stoves }(n=200)\end{array}$ & 120 & Health \\
\hline $\begin{array}{l}\text { Zhou Y, et al. } \\
2014\end{array}$ & China & $\begin{array}{l}996 \text { participants, aged }>40 \text { years, } \\
\text { household }\end{array}$ & $\begin{array}{l}\text { CSS (comparison } \\
\text { between } 2 \text { groups) }\end{array}$ & $\begin{array}{l}\text { I: Biogas digester and improved kitchen ventilation } \\
(n=740) \\
\text { C: Traditional biomass stove (usual practice) }(n=N R)\end{array}$ & 108 & Air quality, health \\
\hline Zuk M, et al. 2007 & Mexico & 53 household & CSS (pre and post) & I: Improved cook stoves (Patsari stoves) & 5 & Air quality \\
\hline
\end{tabular}

$R C T$ randomised control trial, CSS cross sectional study, NR not reported 
Table 4 Characteristics of water quality intervention studies

\begin{tabular}{|c|c|c|c|c|c|c|}
\hline $\begin{array}{l}\text { Study (Author and } \\
\text { publication year) }\end{array}$ & Country & $\begin{array}{l}\text { Participants } \\
\text { (sample size, age, setting) }\end{array}$ & Study design & $\begin{array}{l}\text { Intervention details } \\
(\mathrm{I}=\text { Intervention and } \mathrm{C}=\text { Control })\end{array}$ & $\begin{array}{l}\text { Duration of intervention } \\
\text { (months) }\end{array}$ & Outcome measured \\
\hline Boisson S, et al. 2010 & $\begin{array}{l}\text { Democratic } \\
\text { Republic of } \\
\text { Congo } \\
\text { (DRC) }\end{array}$ & $\begin{array}{l}240 \text { household }(1,144 \\
\text { participants mean age } \\
39.1 \text { years) }\end{array}$ & $\mathrm{RCT}$ & $\begin{array}{l}\text { I: Lifestraw family filter ( } n=120 \text { households, } 546 \\
\text { participants) } \\
\text { C: Placebo filter ( } n=120 \text { households, 598participants) }\end{array}$ & 15 & $\begin{array}{l}\text { Microbial contamination, } \\
\text { health }\end{array}$ \\
\hline Boisson S, et al. 2009 & Ethiopia & $\begin{array}{l}313 \text { households, } \\
6 \text { months and over, } \\
\text { household }\end{array}$ & RCT & $\begin{array}{l}\text { I: Life straw personal filter to be used for ingesting of } \\
\text { untreated water both at home and away from home } \\
(n=155) \\
\text { C: Usual practice }(n=158)\end{array}$ & 5 & $\begin{array}{l}\text { Microbial contamination, } \\
\text { health }\end{array}$ \\
\hline Boisson S, et al. 2013 & India & $\begin{array}{l}2,163 \text { household }(2,986 \\
\text { children }<5 \text { years })\end{array}$ & $\mathrm{RCT}$ & $\begin{array}{l}\text { I: NaDC tablets }{ }^{\mathrm{b}}(n=1080) \\
\text { C: Placebo }(n=1083)\end{array}$ & 12 & $\begin{array}{l}\text { Microbial contamination, } \\
\text { health }\end{array}$ \\
\hline Brown J et al. 2008 & Cambodia & $\begin{array}{l}180 \text { households, all age } \\
\text { participants }\end{array}$ & RCT & $\begin{array}{l}\text { I: One of following: Ceramic water purifier (CWP) } \\
(n=60) \text { and Iron-rich ceramic water purifier (CWP-fe) } \\
(n=60) \\
\text { C: Usual practice }(n=60)\end{array}$ & 5.5 & $\begin{array}{l}\text { Microbial contamination, } \\
\text { health }\end{array}$ \\
\hline Clasen T.F et al. 2006 & Bolivia & $\begin{array}{l}60 \text { households ( } 317 \\
\text { individuals), all age, } \\
\text { household }\end{array}$ & RCT & $\begin{array}{l}\text { l: Water purification filter ( } 20 \text { households; } 210 \text { individuals) } \\
\text { C: Usual practice ( } 40 \text { households; } 107 \text { individuals) }\end{array}$ & 5 & $\begin{array}{l}\text { Microbial contamination, } \\
\text { health }\end{array}$ \\
\hline Clasen T, et al. 2007 & Bangladesh & $\begin{array}{l}100 \text { households, } 555 \\
\text { participants of any age } \\
\text { group }\end{array}$ & RCT & $\begin{array}{l}\text { I: } 67-m g \text { NADCC tablets }{ }^{\mathrm{b}} \text { designed to treat } 20-25 \mathrm{~L} \text { of } \\
\text { water ( } \mathrm{n}=50 \text { households; } 279 \text { participants) } \\
\text { C: Placebo consisting of tablets of the same colour, size } \\
\text { and packaging ( } n=50 \text { households, } 276 \text { participants) }\end{array}$ & 4 & Microbial contamination \\
\hline Clasen T, et al. 2005 & Columbia & 140 household & RCT & $\begin{array}{l}\text { I: Ceramic Water filter ( } n=76 \text { households, } 415 \text { participants) } \\
\text { C: Usual practice ( } n=64 \text { households, } 265 \text { participants) }\end{array}$ & 6 & $\begin{array}{l}\text { Microbial contamination, } \\
\text { health }\end{array}$ \\
\hline $\begin{array}{l}\text { Christen A, } \\
\text { et al. } 2009\end{array}$ & Bolivia & $\begin{array}{l}2 \text { household ( } 27 \text { proxy } \\
\text { household for air quality) }\end{array}$ & $\begin{array}{l}\text { CSS } \\
\text { (pre and post) }\end{array}$ & I: Water disinfection stove (WADIS) & 6 & $\begin{array}{l}\text { Water quality, Microbial } \\
\text { contamination, air quality, } \\
\text { health }\end{array}$ \\
\hline Conroy R, et al. 1996 & Kenya & $\begin{array}{l}206 \text { children age } 5-16 \\
\text { years, household }\end{array}$ & RCT & $\begin{array}{l}\text { I: SODIS bottle }(n=108) \\
\text { C: Only water bottle and suggested to use indoor } \\
(n=98)\end{array}$ & 3 & Health \\
\hline Crump JA, et al. 2005 & Kenya & $\begin{array}{l}605 \text { households ( } 6650 \\
\text { participants) }\end{array}$ & Cluster- RCT & $\begin{array}{l}\text { 11: Flocculant- disinfectant intervention ( } n=201 \\
\text { households,2124 participants) } \\
\text { 12: Sodium hypochlorite intervention ( } n=203 \text { households, } \\
2249 \text { participants) } \\
\text { C: Usual practice ( } n=201 \text { households, } 2277 \text { participants) }\end{array}$ & 4 (20 weeks) & $\begin{array}{l}\text { Microbial contamination, } \\
\text { health }\end{array}$ \\
\hline Davis J, et al. 2011 & Tanzania & $\begin{array}{l}248 \text { households, } \\
\text { participants aged } \\
<5 \text { years }\end{array}$ & $\begin{array}{l}\text { Experimental } \\
\text { field study }\end{array}$ & $\begin{array}{l}\text { I: One of following } 4 \text { intervention: } 1) \text { Information on } \\
\text { strategies to reduce water and sanitation related illness } \\
(n=79) 2) \text { Information as per } 1 \text { plus water quality tests } \\
(n=84) \text { 3) Information as per } 1 \text { plus hand-rinse test } \\
\text { results }(n=90) 4) \text { information as per } 1 \text { plus water and } \\
\text { hand rinse results }(n=81)\end{array}$ & 4 & $\begin{array}{l}\text { Microbial contamination, } \\
\text { hygiene and sanitation }\end{array}$ \\
\hline
\end{tabular}


Table 4 Characteristics of water quality intervention studies (Continued)

\begin{tabular}{|c|c|c|c|c|c|c|}
\hline $\begin{array}{l}\text { Du Preez M, } \\
\text { et al. } 2008\end{array}$ & $\begin{array}{l}\text { Zimbabwe } \\
\text { and South } \\
\text { Africa }\end{array}$ & $\begin{array}{l}115 \text { households, } \\
\text { participants aged } \\
\text { between } 12 \text { to } \\
24 \text { months }\end{array}$ & $\mathrm{RCT}$ & $\begin{array}{l}\text { I: Ceramic water filter }(n=60) \\
\text { C: In-house water filter }(n=58)\end{array}$ & 6 & Health \\
\hline $\begin{array}{l}\text { Du Preez M, } \\
\text { et al. } 2010\end{array}$ & South Africa & $\begin{array}{l}649 \text { households, } \\
6 \text { months to } 5 \text { years, } \\
\text { household }\end{array}$ & $\mathrm{RCT}$ & $\begin{array}{l}\text { I: SODIS bottles to be used to provide drinking } \\
\text { water at all times and as much as possible drink } \\
\text { directly from the bottle }(n=297) \\
\text { C: Usual practice }(n=267)\end{array}$ & 12 & $\begin{array}{l}\text { Microbial contamination, } \\
\text { health }\end{array}$ \\
\hline $\begin{array}{l}\text { Fabiszewski de Aceituno AM, } \\
\text { et al. } 2012\end{array}$ & Honduras & $\begin{array}{l}195 \text { participants aged } \\
<5 \text { years, household }\end{array}$ & $\mathrm{RCT}$ & $\begin{array}{l}\text { I: Plastic Bio sand filters, a narrow mouth gallon } \\
(20 \mathrm{~L}) \text {, water jug and general education on hygiene } \\
\text { and sanitation ( } n=90 \text { households, } 532 \text { participants) } \\
\text { C: Usual practice ( } n=86 \text { households, } 488 \text { participants) }\end{array}$ & 10 & $\begin{array}{l}\text { Microbial contamination, } \\
\text { health }\end{array}$ \\
\hline Graf J, et al. 2010 & Cameroon & $\begin{array}{l}2,193 \text { households, } \\
\text { participants aged } \\
<5 \text { years }\end{array}$ & $\begin{array}{l}\text { CSS (pre and } \\
\text { post) }\end{array}$ & I: SODIS bottles for water purification & 10 & Health \\
\hline Garrett V, et al. 2008 & Kenya & $\begin{array}{l}555 \text { households ( } 960 \\
\text { children aged }<5 \text { years) }\end{array}$ & $\mathrm{RCT}$ & $\begin{array}{l}\text { I: Sodium hypochlorite water disinfection solution } \\
\text { and storage containers and hygiene and sanitation } \\
\text { education }(n=366) \\
\text { C: Usual practice }(n=189)\end{array}$ & 2 (8 weeks) & $\begin{array}{l}\text { Microbial contamination, } \\
\text { health }\end{array}$ \\
\hline Habib MA, et al. 2013 & Pakistan & $\begin{array}{l}18,244 \text {, participants, } \\
\text { household }\end{array}$ & Cluster-RCT & $\begin{array}{l}\text { I: Diarrhoea pack (two packets of low osmolality ORS, } \\
\text { one strip of Zinc tablets, two packets of water purification } \\
\text { sachet and a leaflet with educational materials) }(n=9,581) \\
\text { C: Usual practice }(n=8,663)\end{array}$ & 12 & Health \\
\hline Henry FJ et al. 1990 & Bangladesh & $\begin{array}{l}44 \text { children, } 6-23 \\
\text { months, community }\end{array}$ & $\begin{array}{l}\text { Intervention } \\
\text { Study }\end{array}$ & $\begin{array}{l}\text { I: Latrine construction and hygiene education }(n=41) \\
\text { C: Usual practice }(n=43)\end{array}$ & 6 & Health \\
\hline Henry FJ et al. 1990 & Bangladesh & $\begin{array}{l}92 \text { participants, } 6-18 \\
\text { months, household }\end{array}$ & $\begin{array}{l}\text { Intervention } \\
\text { study }\end{array}$ & $\begin{array}{l}\text { I: Hand pumps, latrine construction and hygiene education } \\
\text { (44) } \\
\text { C: Hand pumps only (48) }\end{array}$ & 6 & Health \\
\hline $\begin{array}{l}\text { Lindquist ED, } \\
\text { et. al; } 2014\end{array}$ & Bolivia & $\begin{array}{l}1,198 \text { participants, } \\
\text { household }\end{array}$ & Cluster-RCT & $\begin{array}{l}\text { 11: A household level hollow fiber filter }(n=330) \\
\text { 12: Education (behaviour change communication) }(n=302) \\
\text { 13: Filter and education }(n=285) \\
\text { C: Life skills and attitudes and family responsibility message } \\
(n=279)\end{array}$ & 3 & Health \\
\hline Luby,AP, et al. 2006 & Pakistan & $\begin{array}{l}1340 \text { households, all age } \\
\text { participants }\end{array}$ & $\mathrm{RCT}$ & $\begin{array}{l}\text { I: One of following intervention: 1) diluted bleach and a } \\
\text { water vessel provided }(n=265) \text { 2) soap and hand washing } \\
\text { promotion provided }(n=262) 3) \text { flocculent disinfectant } \\
\text { water treatment and water vessel provided }(n=262) 4) \\
\text { flocculent-disinfection, soap and hand washing promotion } \\
\text { provided ( } n=266) \\
\text { C: Usual practice }(n=282)\end{array}$ & 9 & Health \\
\hline $\begin{array}{l}\text { Mausezahi D } \\
\text { et al. } 2009\end{array}$ & Bolivia & $\begin{array}{l}484 \text { households, } \\
\text { participants aged } \\
<5 \text { years }\end{array}$ & $\mathrm{RCT}$ & $\begin{array}{l}\text { I: SODIS bottles ( } n=255 \text { households; } 376 \text { children) } \\
\text { C: Usual practice ( } n=200 \text { households; } 349 \text { children) }\end{array}$ & 14 & Health \\
\hline
\end{tabular}


Table 4 Characteristics of water quality intervention studies (Continued)

\begin{tabular}{|c|c|c|c|c|c|c|}
\hline Opryszko MC et al. 2010 & Afghanistan & $\begin{array}{l}1514 \text { households, all age } \\
\text { participants, household }\end{array}$ & $\mathrm{RCT}$ & $\begin{array}{l}\text { I: Multiple intervention; liquid chlorine with a water vessel } \\
\text { ( } 299 \text { households), hygiene education ( } 233 \text { households), } \\
\text { improved tube well ( } 308 \text { households) and combination } \\
\text { of all ( } 261 \text { households) } \\
\text { C: Usual practice }(n=292)\end{array}$ & 17 & Diarrhoeal incidence \\
\hline Quick RE et al. 1996 & Bolivia & 42 household & $\begin{array}{l}\text { Intervention } \\
\text { study (pre } \\
\text { and post) }\end{array}$ & $\begin{array}{l}\text { 11: } 20 \text { I narrow mouthed water vessel and the calcium } \\
\text { hypochlorite solution }(n=15) \\
\text { I2: } 20 \text { I narrow mouthed water vessel }(n=15) \\
\text { C: Usual practice }(n=12)\end{array}$ & 9 weeks & Microbial contamination, \\
\hline Quick RE, et al. 1998 & Bolivia & 127 households & $\mathrm{RCT}$ & $\begin{array}{l}\text { I: Water disinfection solution and storage vessels } \\
\text { ( } n=64 \text { households, } 400 \text { individuals) } \\
\text { C: Usual practice ( } n=63 \text { households, } 391 \text { individuals) }\end{array}$ & 8 & $\begin{array}{l}\text { Microbial contamination, } \\
\text { health }\end{array}$ \\
\hline Ram PK, et al. 2007 & Madagascar & $\begin{array}{l}242 \text { households, } \\
\text { participants aged } \\
\text { 0-90 year }\end{array}$ & $\begin{array}{l}\text { Intervention } \\
\text { study }\end{array}$ & I: Water chlorination tablet and Jerrycan for water storage & NR & $\begin{array}{l}\text { Education and self- } \\
\text { reported compliance }\end{array}$ \\
\hline Rangel JM, et al. 2003 & Guatemala & 100 households & $\mathrm{RCT}$ & $\begin{array}{l}\text { 11: Chlorine bleach and } 20 \text { I narrow mouthed water vessel } \\
(n=20) \\
\text { 12: Combined product }{ }^{c} \text { in narrow mouthed water vessel } \\
(n=20) \\
\text { 13: Combined product }{ }^{c} \text { with customised vessel }(n=20) \\
\text { 14: Combined product }{ }^{c} \text { in traditional vessel }(n=20) \\
\text { C: Traditional vessel }(n=20)\end{array}$ & 1 (4 weeks) & $\begin{array}{l}\text { Microbial contamination, } \\
\text { health }\end{array}$ \\
\hline Rose A et al. 2006 & India & $\begin{array}{l}200 \text { children, } \\
\text { participants aged } \\
<5 \text { years, household }\end{array}$ & $\mathrm{RCT}$ & $\begin{array}{l}\text { I: SODIS bottles for water purification plus diarrhoea } \\
\text { prevention and treatment education }(n=100) \\
\text { C: Diarrhoeal prevention and treatment education only } \\
(n=100)\end{array}$ & 6 & Health \\
\hline Rosa G, et al. 2014 & Rwanda & 566 households & $\mathrm{RCT}$ & $\begin{array}{l}\text { I: Life straw family } 2.0 \text { filter and one improved stove } \\
\text { (Eco Zoom Dura) }(n=285) \\
\text { C: Usual practice }(n=281)\end{array}$ & 5 & Water quality, air quality \\
\hline Stauber CE, et al. 2009 & $\begin{array}{l}\text { Dominican } \\
\text { Republic }\end{array}$ & $\begin{array}{l}187 \text { households, } \\
\text { all aged participants }\end{array}$ & $\mathrm{RCT}$ & $\begin{array}{l}\text { I: Plastic Bio Sand filters ( } n=81 \text { households, } 447 \\
\text { participants) } \\
\text { C: Usual practice ( } n=86 \text { households, } 460 \text { participants) }\end{array}$ & 10 & $\begin{array}{l}\text { Microbial contamination, } \\
\text { health }\end{array}$ \\
\hline Stauber CE, et al. 2011 & Cambodia & $\begin{array}{l}189 \text { households, } \\
\text { participants aged } \\
<5 \text { years }\end{array}$ & $\mathrm{RCT}$ & $\begin{array}{l}\text { I: Plastic Bio Sand filters ( } n=90 \text { households, } 546 \\
\text { participants) } \\
\text { C: Usual practice ( } n=99 \text { households, } 501 \text { participants) }\end{array}$ & 6 & $\begin{array}{l}\text { Microbial contamination, } \\
\text { health }\end{array}$ \\
\hline Tiwari SS, et al. 2009 & Kenya & 59 household & $\mathrm{RCT}$ & $\begin{array}{l}\text { I: Concrete Bio sand Filter and instruction on filter use } \\
(n=30) \\
\text { C: Usual practice }(n=29)\end{array}$ & 6 & $\begin{array}{l}\text { Microbial contamination, } \\
\text { health }\end{array}$ \\
\hline
\end{tabular}

$R C T$ randomised control trial, CSS cross sectional study, NR not reported, a SODIS: Solar Disinfection method, ${ }^{\mathrm{b}}$ NADCC tablets: Sodium Dichloroisocyanurate tablets, ${ }^{\mathrm{C}}$ Combined product: a product incorporating precipitation, coagulation, flocculation and chlorination technology 
10 in Asia and the remaining 13 in African countries. All of these studies were conducted in household settings.

Twenty-seven of these studies looked at the impact of intervention on health especially on the incidence/prevalence of diarrhoeal diseases; 20 on microbial contaminations and water quality; two studies examined the level of knowledge and self-compliance, two investigated air quality and one hygiene and sanitation.

\section{Characteristics of nutrition Interventions $(n=30)$}

Of the 30 nutrition intervention studies included in the review (Table 5), 11 studies were supplementary food and vitamin interventions, 13 nutrition education interventions, five nutrition education together with complementary food interventions, two combined interventions of nutrition education and home gardening [33, 34] and one combined package intervention of health care, nutrition education, water and sanitation [35].

Most of the studies $(n=18)$ were intervention studies (pre and post or two group comparison), ten RCT, one randomised crossover study and one crossover trial. The sample sizes of the studies ranged from 42 [36] to 40,000 [37] participants. The duration of the study also varied; from a once-off nutrition counselling training [38] to a 48 months nutrition education intervention in Nicaragua [39]. Just over half of the studies $(n=16)$ were conducted in Asia, nine in Africa and the other six in South American countries. Majority of these studies $(n=17)$ were conducted in a household settings with some in community settings.

Eighteen of the nutrition intervention studies assessed the impact of intervention on nutritional status such as growth, prevalence of stunting (low height-for-age), underweight (low weight-for-age), and wasting (low weight-for-height), 10 studies assessed food consumption and dietary diversity, nine studies assessed the impact on nutrient deficiencies, eight studies looked at health status, six at nutrient intake, five at health and nutritional knowledge, two at feeding practice and one assessed food security.

\section{Methodology quality}

Of the 123 included studies, eight studies failed to provide sufficient detail to assess their methodological quality. Information of study selection, withdrawals, blinding and confounders were particularly under-reported in the majority of studies. Because of the nature of the intervention, it was assumed that no blinding was imposed in some studies and they were therefore categorised into moderate quality study. The most common methodological problems among the weak studies were in selection bias, confounders, reliability and validity of data collection tools and blinding.

\section{Discussion}

According to our knowledge, this systematic review is the first to explore the cross-domain overlapping of multidisciplinary research projects in agriculture, nutrition, air quality and water quality. It is obvious that there is a lot of work being done in this area but from this review it clear that there is variation in not only the type of intervention, study type, sample size, duration and setting, but also in the outcome measured.

Although a wide variety of agricultural interventions such as home gardening and animal husbandry were conducted to improve household food productivity and food consumption, this review also confirms the findings of previous reviews that only few studies were measuring the impact of those interventions on nutritional status [8-10]. Of those projects that did look at the impact of agricultural intervention on nutrition, seven examined the impact on nutrient intake, nutrient deficiencies and anthropometry. In general it is predictable that increased production and consumption of food leads to better nutrition, but due to variation in study design, duration and outcome of interest measured among the included studies, it doesn't look likely to obtain pooled estimate for studies which look at impact of intervention on nutritional health.

While looking at the air quality interventions, it is evident that interventions to improve cook stoves are the most popular interventions ( $83 \%$ ) and are widely being used in all over the world. This may provide the enough roofs to perform the meta-analysis. Some biogas interventions $(n=4)$ [20, 40-42] have been conducted to measure the multiple benefits of intervention on indoor air quality and food production (using bio-slurry). However, as they refer to different outcome measures and are measured in different ways, the available evidence does not look strong enough to perform the comprehensive analysis.

It was identified that water purification filter interventions were the most popular $(n=12)$ interventions for treatment of drinking water quality in LMIC. Other interventions such as chlorine tablets or solution $(n=9)$ and solar disinfection $(n=7)$ are also common in this region. Randomised controlled trial study design was the most popular among the water quality intervention as the vast majority (78 \%) of the research project applied this method. So, it is more likely that effects on the drinking water quality can be summarised across studies.

Nutrition education $(n=13)$ and supplementary food and vitamin $(n=11)$ interventions were the most popular nutritional intervention in LMIC. Some intra-domain combined interventions of nutrition education and supplementary foods $(n=5)$ have also been piloted in some low and middle income countries to determine the 
Table 5 Characteristics of nutrition intervention studies

\begin{tabular}{|c|c|c|c|c|c|c|}
\hline $\begin{array}{l}\text { Study (Author and } \\
\text { publication year) }\end{array}$ & Country & $\begin{array}{l}\text { Participants } \\
\text { (sample size, age, setting) }\end{array}$ & Study design & $\begin{array}{l}\text { Intervention details } \\
(\mathrm{I}=\text { Intervention and } \mathrm{C}=\text { Control) }\end{array}$ & $\begin{array}{l}\text { Duration of intervention } \\
\text { (months) }\end{array}$ & Outcome measured \\
\hline Ali D et al. 2013 & $\begin{array}{l}\text { Bangladesh, } \\
\text { Vietnam, } \\
\text { Ethiopia }\end{array}$ & $\begin{array}{l}2356 \text { (Ethiopia), } 3075 \text { (Vietnam), } \\
3422 \text { (Bangladesh) households, } \\
\text { participants aged } 6 \\
\text { monthsnths-5 years }\end{array}$ & CSS & I: Nutrition education & NR & $\begin{array}{l}\text { Food consumption } \\
\text { and anthropometry }\end{array}$ \\
\hline $\begin{array}{l}\text { Chow J, et al. } \\
2010\end{array}$ & India & $\begin{array}{l}\text { participants aged 1-4 years, } \\
\text { household }\end{array}$ & Intervention study & $\begin{array}{l}\text { I: High dose vitamin A supplementation, } \\
\text { Industrial fortification of mustard oil and } \\
\text { GM fortification of mustard oil and seed }\end{array}$ & NR & Health \\
\hline $\begin{array}{l}\text { Creed-Kanashiro } \\
\text { H et al. } 2003\end{array}$ & Peru & $\begin{array}{l}42 \text { participants, aged 12-51 } \\
\text { years, community }\end{array}$ & $\begin{array}{l}\text { Interventional study } \\
\text { (pre and post) }\end{array}$ & I: Nutrition education & $N R$ & $\begin{array}{l}\text { Nutrient deficiencies, } \\
\text { education }\end{array}$ \\
\hline $\begin{array}{l}\text { Darapheak C, } \\
\text { et al. } 2013\end{array}$ & Cambodia & $\begin{array}{l}6202 \text { participants, aged 12-59 } \\
\text { months, household }\end{array}$ & $\begin{array}{l}\text { CSS (post } \\
\text { intervention only) }\end{array}$ & $\begin{array}{l}\text { I: Animal source food group } \\
\text { C: Non animal source food group }\end{array}$ & NR & $\begin{array}{l}\text { Anthropometry, } \\
\text { health }\end{array}$ \\
\hline $\begin{array}{l}\text { English RM, et al. } \\
1997\end{array}$ & Vietnam & $\begin{array}{l}720 \text { children }<6 \text { years, } \\
\text { community }\end{array}$ & CSS (2 groups) & $\begin{array}{l}\text { I: Home gardening and nutrition education } \\
(n=469) \\
\text { C: Usual practice }(n=251)\end{array}$ & $24-36$ & $\begin{array}{l}\text { Nutrient intake, } \\
\text { health }\end{array}$ \\
\hline $\begin{array}{l}\text { Faber M, et al. } \\
2002\end{array}$ & South Africa & $\begin{array}{l}208 \text { participants, aged } 2-5 \\
\text { years, community }\end{array}$ & CSS (Pre and post) & $\begin{array}{l}\text { I: Home gardening along with nutrition education } \\
(n=108) \\
\text { C: Usual practice }(n=100)\end{array}$ & 20 & Nutrient intake \\
\hline Fenn B et al. 2012 & Ethiopia & $\begin{array}{l}5552 \text { participants, } 6-36 \\
\text { monthsnths, household }\end{array}$ & CSS (pre and post) & $\begin{array}{l}\text { I: Multiple intervention; health care, nutrition } \\
\text { education, water and sanitation ( } 4124) \\
\text { C: Protective safety net programme (1428) }\end{array}$ & 30 & Anthropometry \\
\hline $\begin{array}{l}\text { Gibson RS et al. } \\
2003\end{array}$ & Malawi & $\begin{array}{l}281 \text { participants, aged between } \\
30-40 \text { months, household }\end{array}$ & $\begin{array}{l}\text { Quasi- } \\
\text { experimental }\end{array}$ & $\begin{array}{l}\text { I: Complementary foods }(n=200) \\
\text { C: Usual practice }(n=81)\end{array}$ & 6 & $\begin{array}{l}\text { Food consumption, } \\
\text { nutrient intake, } \\
\text { anthropometry }\end{array}$ \\
\hline $\begin{array}{l}\text { Grillenberger, } \\
\text { et al. } 2006\end{array}$ & Kenya & $\begin{array}{l}498 \text { participants, mean age } \\
7.4 \text { years }\end{array}$ & RCT & $\begin{array}{l}\text { I: Three supplementary foods groups: meat }(n=134) \text {, } \\
\text { milk }(n=144) \text { and energy (veg oil) supplied as a school } \\
\text { snack in a maize stew ( } n=148) \\
\text { C: Usual practice }(n=129)\end{array}$ & 24 & Anthropometry \\
\hline $\begin{array}{l}\text { Grillenberger, } \\
\text { et al. } 2006\end{array}$ & Kenya & $\begin{array}{l}554 \text { participants, mean age } \\
7.4 \text { years }\end{array}$ & RCT & $\begin{array}{l}\text { I: Three supplementary foods groups: meat }(n=134) \\
\text { milk }(n=144) \text { and energy (veg oil) supplied as a school } \\
\text { snack in a maize stew }(n=148) \\
\text { C: Usual practice }(n=129)\end{array}$ & 24 & $\begin{array}{l}\text { Nutrient intake, } \\
\text { anthropometry }\end{array}$ \\
\hline $\begin{array}{l}\text { Imran M, et al. } \\
2014\end{array}$ & India & $\begin{array}{l}245 \text { participants, aged } 2-4 \\
\text { years, community }\end{array}$ & Intervention study & $\begin{array}{l}\text { I: Nutrition education along with supplementary } \\
\text { nutrition and supervision }\end{array}$ & 12 & Anthropometry \\
\hline $\begin{array}{l}\text { Kabahenda M, et } \\
\text { al. } 2011\end{array}$ & Uganda & $\begin{array}{l}89 \text { children }<4 \text { years, } \\
\text { household }\end{array}$ & RCT & $\begin{array}{l}\text { I: Nutrition education }(n=46) \\
\text { C: Sewing classes }(n=43)\end{array}$ & 12 & $\begin{array}{l}\text { Food consumption, } \\
\text { nutrient deficiencies }\end{array}$ \\
\hline $\begin{array}{l}\text { Khan A Z et al. } \\
2013\end{array}$ & Pakistan & $\begin{array}{l}586 \text { participants, aged } 6 \text { mo- } \\
8 \text { years, household }\end{array}$ & $\begin{array}{l}\text { Intervention study } \\
\text { (pre and post) }\end{array}$ & I: Nutrition education & 3 & $\begin{array}{l}\text { Food consumption, } \\
\text { anthropometry }\end{array}$ \\
\hline $\begin{array}{l}\text { Kilaru A, et al. } \\
2005\end{array}$ & India & $\begin{array}{l}242 \text { infants aged } 5-11 \text { months, } \\
\text { household }\end{array}$ & Intervention study & $\begin{array}{l}\text { I: Nutrition education ( } n=173) \\
\text { C: No nutrition education }(n=69)\end{array}$ & 36 & $\begin{array}{l}\text { Food consumption, } \\
\text { Anthropometry }\end{array}$ \\
\hline $\begin{array}{l}\text { Lanerolle P and } \\
\text { Atukorala S, } 2006\end{array}$ & Sir Lanka & $\begin{array}{l}229 \text { adolescent girls aged } \\
\text { between } 15-19 \text { years, } \\
\text { household }\end{array}$ & $\begin{array}{l}\text { Intervention study } \\
\text { (pre and post) }\end{array}$ & I: Nutrition education & 10 weeks & $\begin{array}{l}\text { Nutrition } \\
\text { knowledge, food }\end{array}$ \\
\hline
\end{tabular}


Table 5 Characteristics of nutrition intervention studies (Continued)

\begin{tabular}{|c|c|c|c|c|c|c|}
\hline $\begin{array}{l}\text { Moore JB, et al. } \\
2009\end{array}$ & Nicaragua & $\begin{array}{l}182 \text { adolescents and } 67 \\
\text { mothers, community }\end{array}$ & $\begin{array}{l}\text { Longitudinal study } \\
\text { (pre and post) }\end{array}$ & I: Nutrition education & $\begin{array}{l}48 \text { for girls and } 24 \text { for } \\
\text { mothers }\end{array}$ & $\begin{array}{l}\text { Nutritional } \\
\text { knowledge, nutrient } \\
\text { deficiencies }\end{array}$ \\
\hline $\begin{array}{l}\text { Pawloski LR and } \\
\text { Moore JB; } 2007\end{array}$ & Nicaragua & $\begin{array}{l}186 \text { adolescent girls aged } \\
10-17 \text { years, community }\end{array}$ & $\begin{array}{l}\text { Intervention study } \\
\text { (pre and post) }\end{array}$ & I: Nutrition education & 36 & $\begin{array}{l}\text { Nutritional } \\
\text { knowledge, } \\
\text { Anthropometry, } \\
\text { nutrient deficiencies }\end{array}$ \\
\hline $\begin{array}{l}\text { Phawa S, et al. } \\
2010\end{array}$ & India & $\begin{array}{l}370 \text { mothers of children aged } \\
12-71 \text { months, community }\end{array}$ & $\begin{array}{l}\text { Intervention study } \\
\text { (2 groups) }\end{array}$ & $\begin{array}{l}\text { I: Nutrition and health education }(n=195) \\
\text { C: Usual practice }(n=175)\end{array}$ & 9 & Health \\
\hline $\begin{array}{l}\text { Pant CR, et al. } \\
1996\end{array}$ & Nepal & $\begin{array}{l}40,000 \text { children aged } 6-12 \\
\text { months }\end{array}$ & $\begin{array}{l}\text { Intervention study } \\
\text { (pre and post) }\end{array}$ & $\begin{array}{l}\text { I: Mega dose vitamin A capsules and nutrition } \\
\text { education } \\
\text { C: Usual practice }\end{array}$ & 24 & $\begin{array}{l}\text { Health, nutrient } \\
\text { deficiencies }\end{array}$ \\
\hline $\begin{array}{l}\text { Rivera JA, et al. } \\
2004\end{array}$ & Mexico & $\begin{array}{l}650 \text { children aged }<12 \text { months, } \\
\text { household }\end{array}$ & $\begin{array}{l}\text { Randomised } \\
\text { crossover study }\end{array}$ & $\begin{array}{l}\text { I: Nutrition Education along with micronutrient- } \\
\text { fortified foods }(n=373) \\
\text { C: Cross over intervention group }(n=277)\end{array}$ & 24 & $\begin{array}{l}\text { Anthropometry, } \\
\text { nutrient deficiencies }\end{array}$ \\
\hline Roy SK, et al. 2005 & Bangladesh & $\begin{array}{l}282 \text { children aged } 6-24 \\
\text { months, household }\end{array}$ & RCT & $\begin{array}{l}\text { 11: Intensive nutrition education twice a week } \\
\text { 12: Intensive nutrition education and supplementary food } \\
\text { C: Nutrition education from community nutrition promotors }\end{array}$ & 3 & $\begin{array}{l}\text { Food consumption } \\
\text { Anthropometry, } \\
\text { Nutrient intake, } \\
\text { Education }\end{array}$ \\
\hline $\begin{array}{l}\text { Salehi M, et al. } \\
2004\end{array}$ & Iran & $\begin{array}{l}811 \text { children aged }<5 \text { years, } \\
\text { household }\end{array}$ & $\begin{array}{l}\text { Intervention study } \\
\text { (2 groups) }\end{array}$ & $\begin{array}{l}\text { I: Nutrition education }(n=406) \\
\text { C: Usual practice }(n=405)\end{array}$ & 12 & $\begin{array}{l}\text { Anthropometry, } \\
\text { Food consumption }\end{array}$ \\
\hline $\begin{array}{l}\text { Santos l, et al. } \\
2001\end{array}$ & Brazil & $\begin{array}{l}424 \text { participants, aged } \\
<18 \text { months, community }\end{array}$ & $\mathrm{RCT}$ & $\begin{array}{l}\text { I: Nutritional counselling }(n=218) \\
\text { C: Usual practice }(n=206)\end{array}$ & One off training & Anthropometry \\
\hline $\begin{array}{l}\text { Sazawal S, et al. } \\
2010\end{array}$ & India & $\begin{array}{l}633 \text { participants, } 1-4 \text { years, } \\
\text { community }\end{array}$ & RCT & $\begin{array}{l}\text { I: Micronutrient fortified milk }(n=316) \\
\text { C: Non-fortified milk }(n=317)\end{array}$ & 12 & $\begin{array}{l}\text { Anthropometry and } \\
\text { nutrient deficiencies }\end{array}$ \\
\hline $\begin{array}{l}\text { Sekartini R et al. } \\
2013\end{array}$ & Indonesia & $\begin{array}{l}54 \text { participants, aged between } \\
5-6 \text { years, household }\end{array}$ & $\mathrm{RCT}$ & $\begin{array}{l}\text { I: Four different complementary milks products; Std GUM, } \\
\text { Iso-5 GUM, Iso-5 LP GUM, Iso-2.5 GUM }\end{array}$ & 2 & Health \\
\hline $\begin{array}{l}\text { Siekmann JF et al. } \\
2003\end{array}$ & Kenya & $\begin{array}{l}555 \text { participants aged between } \\
5-14 \text { years }\end{array}$ & RCT & $\begin{array}{l}\text { I: Three supplementary foods groups: meat }(n=134) \text {, milk ( } n \\
=144) \text { and energy (veg oil) supplied as a school snack in a } \\
\text { maize stew }(n=148) \\
\text { C: Usual practice }(n=129)\end{array}$ & 12 & $\begin{array}{l}\text { Food consumption, } \\
\text { nutrient intake }\end{array}$ \\
\hline $\begin{array}{l}\text { Serkatini R et al. } \\
2013\end{array}$ & Indonesia & $\begin{array}{l}54 \text { participants, aged } 5-6 \\
\text { years, household }\end{array}$ & Cross over study & $\begin{array}{l}\text { I: Four different growing up milk (GUM) products - Standard } \\
\text { GUM, Std GUM with } 5 \mathrm{~g} \text { isomaltulose per serving (Iso-5 } \\
\text { GUMO, Iso-5 GU with lowered protein content (Iso-5 LP } \\
\text { GUM), Std GUM with } 2.5 \mathrm{~g} \text { isomaltulose in combination with } \\
\text { other vitamins and minerals (Iso } 2.5 \text { GUM) }\end{array}$ & 2 & Health \\
\hline
\end{tabular}

I: One of following complementary fortified foods: $\quad 6 \quad$ Anthropometry

Weanimix (W) a combination of soybeans, maize

and groundnuts, Weanimix plus mineras and

vitamins (WM), Weanimix plus fish powder (WF)

and Koko plus fish powder (KF) $(n=208)$

C: Usual practice $(n=465)$

Lartey A et al. Ghana 216 participants, aged 6-12

months, households

$\mathrm{RCT}$

consumption

nutrient deficiencies

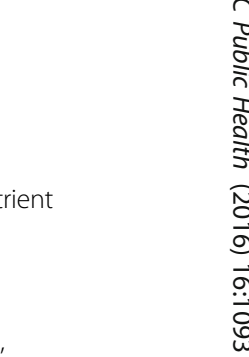


Table 5 Characteristics of nutrition intervention studies (Continued)

\begin{tabular}{|c|c|c|c|c|c|c|}
\hline $\begin{array}{l}\text { Vitolo M R et al. } \\
2008\end{array}$ & Brazil & $\begin{array}{l}500 \text { individuals, all age, } \\
\text { household }\end{array}$ & $\mathrm{RCT}$ & $\begin{array}{l}\text { I: Breastfeeding and weaning counselling and } \\
\text { complementary foods ( } 163 \text { mothers baby pairs) C: No dietary } \\
\text { advice given ( } 234 \text { mother-baby pairs) }\end{array}$ & 6 & Health \\
\hline $\begin{array}{l}\text { Walsh CM, et al. } \\
2002\end{array}$ & South Africa & $\begin{array}{l}815 \text { children aged } 2 \text { to } 5 \text { years, } \\
\text { household }\end{array}$ & $\begin{array}{l}\text { Intervention study } \\
\text { ( } 2 \text { groups) }\end{array}$ & $\begin{array}{l}\text { I: Nutrition education plus food aid } \\
\text { C: Food aid only }\end{array}$ & 24 & $\begin{array}{l}\text { Anthropometry, } \\
\text { nutrient deficiencies }\end{array}$ \\
\hline
\end{tabular}


impact of intervention on dietary diversity and nutrient intake.

The main finding of this review is that the vast majority (91\%) of the academic research on agricultural, nutrition and the environmental studies are simple and discipline specific with substantially fewer $(n=11)$ combined interventions across domains and the result is consistent with previous domain specific reviews [7, 43]. Only six studies looked at the combined impact of agricultural and nutrition education interventions, three on air and water quality interventions, one study examined the impact of a combination of agricultural and air quality interventions and one was a combined water quality and nutritional intervention. Although poor nutrition and household air pollution are the leading cause of mortality in LMIC [3], this review did not find any studies examining the impact of a combination of air quality and nutritional interventions on health. It is also striking that none of these studies investigating the combined impact of agricultural and drinking water quality interventions on human health. The evidence reviewed here shows that silo mentality is still inherent in academic research.

Another interesting finding of this review is that certain LMIC regions seem to focus on domain-specific interventions, with most studies in Kenya and India and only a small number in other countries (Fig. 3). Asian and African countries were the most common regional target for agricultural and nutritional studies. More than half of the agricultural (52\%) and nutritional (53\%) interventions were conducted in Asian countries with the majority of them in south Asian countries. Similarly, $48 \%$ of agricultural and $30 \%$ of nutritional studies were conducted in Africa with the majority of them focussed in sub-Saharan African countries such as Kenya, Ethiopia and South Africa. The majority of water quality interventions were conducted in Africa (40.6\%) followed by Asia (31.3 \%) and Latin America (28.1\%). However, the majority (53\%) of interventions to improve household air quality were conducted in Latin American countries particularly in Guatemala, Peru and Mexico. This restricts the generalisability of the findings to other LMIC.

\section{Strengths and limitations of the study}

The main strength of this review is the application of a comprehensive search strategy through four databases to capture all potentially relevant peer reviewed articles. One hundred and twenty three articles representing the four different intervention domains provide ample evidence to understand the current research gap in interdisciplinary research. The use of independent reviewers throughout the review process further strengthened the methodological quality.

The main limitation of this study is that as only peer reviewed journal articles were included in this review, there is a chance of missing those studies published in developmental organisations' reports and bulletins (publication bias). Additionally, this review focused on household and community-based studies, so there is a chance of missing some useful studies conducted in clinical settings.

\section{Conclusion}

In conclusion, it is evident that very little interdisciplinary research has been conducted with the majority of

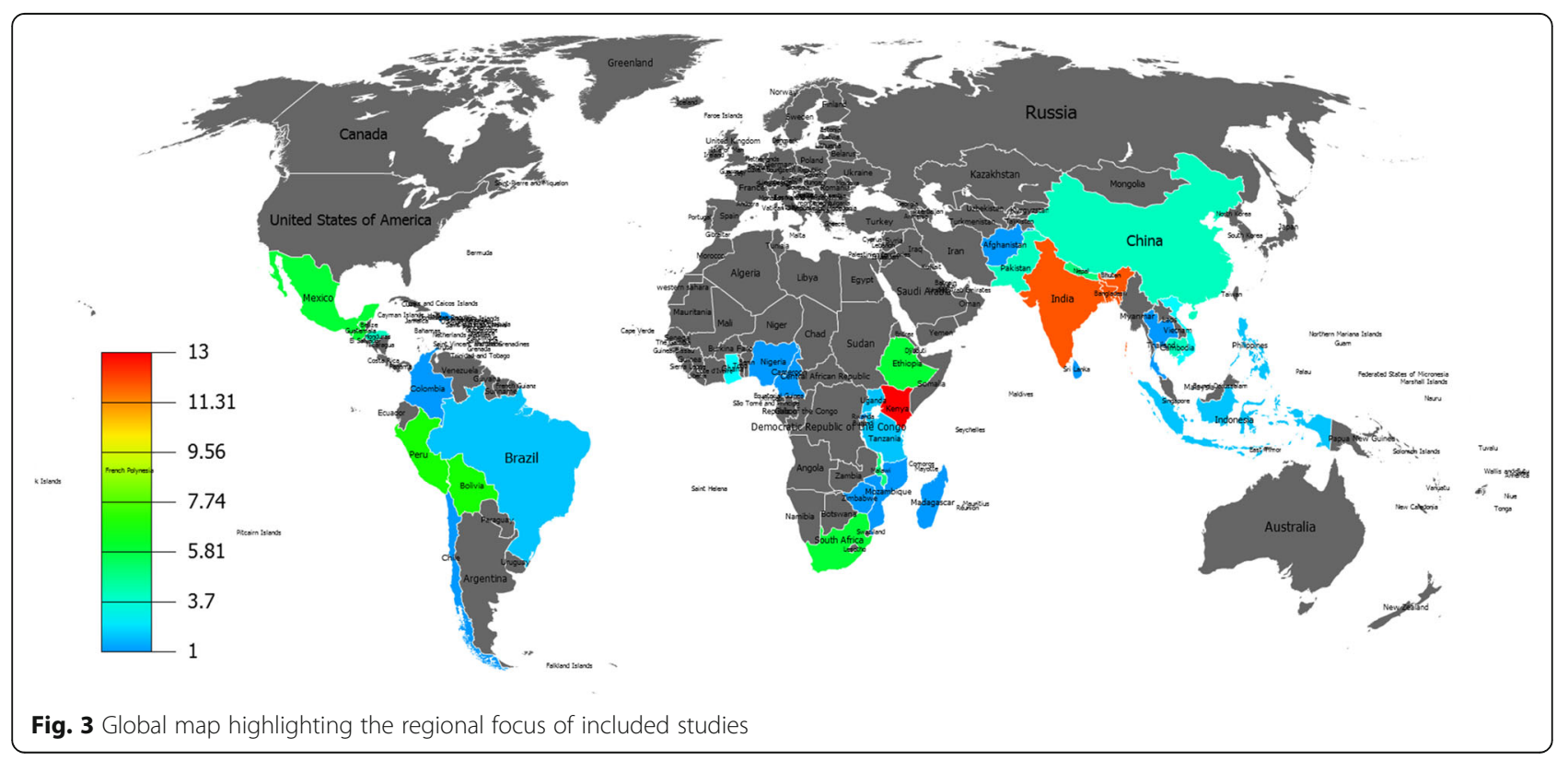


studies on agriculture, nutrition and the environment being discipline specific. It also seems that certain LMIC regions seem to focus on domain-specific interventions. Although a wide variety of study designs have been implemented to measure the impact of agricultural, nutrition and air quality interventions on respective outcomes of interest measured, there is still not sufficient evidence which utilises robust randomised or quasi-experimental study design.

Therefore, this review emphasizes that future research needs to focus on multi-disciplinary complex interventions with standardised outcome measures. Also, rigorous research across disciplines and sharing expertise across regions is a necessity. The next phase of this review (Meta-analysis) will identify whether eliminating silos of discipline specific research can bring a significant improvement or not.

\section{Additional files}

Additional file 1: Ovid Embase Search Strategy. (DOCX $16 \mathrm{~kb}$ )

Additional file 2: Data Extraction Sheet. (DOCX $21 \mathrm{~kb}$ )

\section{Abbreviations}

ARI: Acute respiratory infections; C: Control group; CO: Carbon monoxide; COPD: Chronic obstructive pulmonary disease; CRT: Cluster-randomised trial; CSS: Cross-sectional study; DDS: Dietary diversity score; HAZ: Height for age Zscore; I: Intervention group; LMIC: Low and middle income countries; MeSH: Medical subject heading; NADCC tablets: Sodium dichloroisocyanurate tablets; NR: Not reported; PICOS: Participants, interventions, comparators, outcomes and study design; PM2.5: Fine particulate matter of diameter $<2.5 \mu \mathrm{m}$; PRISMA: Preferred reporting items for systematic reviews and meta-analyses; RCT: Rrandomised control trial; SODIS: Solar disinfection method; TCC: Thermo tolerant coliforms; WAZ: Weight for age Z-score

\section{Acknowledgements}

We are grateful to Dr Steve Turner and Dr Adam Price for their insightful comments that improved the manuscript. We would like to thank Heather Clark and Bimbola Kalejaiye for their help in data extraction. We are also grateful to Melanie Bickerton and Dr Amudha Poobalan for their systematic review advice.

\section{Funding}

The authors have no financial relationships relevant to this article to disclose.

\section{Availability of data and materials}

The datasets supporting the conclusions of this article are included within the article and its additional files.

\section{Authors' contributions}

SG drafted the study protocol, conducted the systematic review and wrote the manuscript. JK, SS, JS, MS and DM contributed to search strategy, assessed the quality of the data extraction process and contributed to the analysis plan and authorship of the manuscript. All authors read and approved the final manuscript.

\section{Competing interests}

The authors declare that they have no competing interests.

\section{Consent for publication}

Not applicable.

Ethics approval and consent to participate Not applicable.

\section{Author details}

'Division of Applied Health Sciences, University of Aberdeen, Room 1.019 Polwarth Building, Foresterhill, Aberdeen AB25 2ZD, UK. ${ }^{2}$ School of Biological Sciences, University of Aberdeen, Aberdeen, UK. ${ }^{3}$ Save the Children, Kathmandu, Nepal. "WMS- Health Education, The University of Warwick, Coventry, UK.

Received: 23 February 2016 Accepted: 29 September 2016

Published online: 18 October 2016

\section{References}

1. The State of Food Insecurity in the World. Strengthening the enabling environment for food security and nutrition, UN Food and Agricultural Organisation (FAO), 2014.

2. Global Food security index 2015: An annual measure of the state of global food security. The Economist Group, 2015.

3. Lim SS, Vos T, Flaxman AD, et al. A comparative risk assessment of burden of disease and injury attributable to 67 risk factors and risk factor clusters in 21 regions, 1990-2010: a systematic analysis for the Global Burden of Disease Study 2010. Lancet. 2012;380:2224-60.

4. Kurmi OP, Semple S, Steiner M, Henderson GD, Ayres JG. Particulate matter exposure during domestic work in Nepal. Ann Occup Hyg. 2008;52:509-17.

5. Kurmi OP, Gaihre S, Semple S, Ayres JG. Acute exposure to biomass smoke causes oxygen desaturation in adult women. Thorax. 2011;66:724-5.

6. Ezzati M, Kammen DM. Quantifying the Effects of Exposure to Indoor Air Pollution from Biomass Combustion on Acute Respiratory Infections in Developing Countries. Environ Health Perspect. 2001;109:481-8.

7. UNICEF. Strategy for Improved Nutrition of Children and Women in Developing Countries. New York: UNICEF; 1990. p. 18-23.

8. Masset E, Haddad L, Cornelius A, Isaza-Castro J. Effectiveness of agricultural interventions that aim to improve nutritional status of children: systematic review. BMJ. 2012;344:d8222.

9. Berti PR, Krasevec J, FitzGerald S. A review of the effectiveness of agriculture interventions in improving nutrition outcomes. Public Health Nutr. 2004;7:599-609.

10. Ruel MT. Can Food-Based Strategies Help Reduce Vitamin A and Iron Deficiencies? A Review of Recent Evidence. Washington, DC: IFPRI (International Food Policy Research Institute); 2001.

11. Rehfuess EA, Puzzolo E, Stanistreet D, Pope D, Bruce NG. Enablers and Barriers to Large-Scale Uptake of Improved Solid Fuel Stoves: A Systematic Review. Environ Health Perspect. 2014;122:120-30.

12. Moher D, Liberati A, Tetzlaff J, Altman DG. Preferred reporting items for systematic reviews and meta-analyses: the PRISMA statement. BMJ. 2009; 339:b2535.

13. Higgins JPT, Green S. Cochrane handbook for systematic reviews of interventions: Cochrane book series. 2009.

14. Jackson N, Waters E. Guidelines for Systematic Reviews of Health Promotion and Public Health Interventions Taskforce. Guidelines for Cochrane systematic reviews of public health interventions. Health Promot Int. 2005;20:367-74.

15. Hotz C, Loechl C, et.al. Introduction of b-Carotene-Rich Orange Sweet Potato in Rural Uganda Resulted in Increased Vitamin A Intakes among Children and Women and Improved Vitamin A Status among Children. The Journal of Nutrition. 2012. p.1871-1880.

16. Yakubu A, Ari MM, Ogbe1 AO, Ogah DM, Adua MM, Idahor KO, Alu SE, Ishaq AS and Salau ES. Preliminary investigation on community-based intervention through cockerel exchange programme for sustainable improved rural chicken production in Nasarawa State, Nigeria. Livestock Research for Rural Development. 2014, 26. http://www.Irrd.cipav.org.co/ Irrd26/10/yaku26184.htm.

17. Wyatt AJ, Yount KM, Null C, Ramakrishnan U, Girard AW. Dairy intensification, mothers and children: an exploration of infant and young child feeding practices among rural dairy farmers in Kenya. Maternal Child Nutr. 2015;11:88-103.

18. Schmid M, Salomeyesudas B, Satheesh P, Hanley J, Kuhnlein HV. Intervention with Traditional Food as a Major Source of Energy, Protein, Iron, Vitamin C, and Vitamin A for Rural Dalit Mothers and Young Children in Andhra Pradesh, South India. Asia Pac J Clin Nutr. 2007;16(1):84-93.

19. Bolem MW, Huq N, Gorstein J, Burger S, Kahn T, Islam N, et al. Production of fruits and vegetables at the homestead is an important source of Vitamin A among women in rural Bangladesh. Eur J Clin Nutr. 1996;50S:62-7. 
20. Chowdhury Z, Le LT, Masud AA, Chang KC, Alauddin M, Hossain M, et al. Quantification of indoor air pollution from using cookstoves and estimation of its health effects on adult women in Northwest Bangladesh. Aerosol Air Qual Res. 2012;12(4):463-75.

21. Hartinger SM, Lanata CF, Gil Al, Hattendorf J, Verastegui H, Mausezahl D. Combining interventions: improved chimney stoves, kitchen sinks and solar disinfection of drinking water and kitchen clothes to improve home hygiene in rural Peru. J Field Actions. 2012;6:1-11.

22. McCracken JP, Smith KR. Emissions and efficiency of improved woodburning cookstoves in Highland Gatemala. Environ Int. 1998;24(7):739-47.

23. Harris SA, Weeks JB, Chen JP, Layde P. Health effects of an efficient vented stove in the highlands of Guatemala. Glob Public Health. 2011;6(4):421-32.

24. Li Z, Mulholland JA, Romanoff LC, Pittman EN, Trinidad DA, Lewin MD, et al. Evaluation of exposure reduction to indoor air pollution in stove intervention projects in Peru by urinary biomonitoring of polycyclic aromatic hydrocarbon metabolites. Environ Int. 2011;37(7):1157-63.

25. Pndey MR, Neupane RP, Gautam A, Shrestha B. The Effectiveness of Smokeless Stoves in Reducing Indoor Air Pollution in a Rural Hill Region of Nepal. Mountain Res Develop. 1990;10(4):313-20.

26. Henry FJ, Huttly SRA, Patwary Y, Aziz KMA. Environmental sanitation, food and water contamination and diarrhoea in rural Bangladesh. Epidemiol Infect. 1990;104(02):253-9.

27. de Aceituno AM F, Stauber CE, Walters AR, Meza Sanchez RE, Sobsey MD. A Randomized Controlled Trial of the Plastic-Housing BioSand Filter and Its Impact on Diarrheal Disease in Copan, Honduras. Am J Trop Med Hyg 2012;86(6):913-21.

28. Garrett V, Ogutu P, Mabonga P, Ombeki S, Mwaki A, Aluoch G, Phelan M, Quick RE. Diarrhoea prevention in a high-risk rural Kenyan population through point-of-use chlorination, safe water storage, sanitation, and rainwater harvesting. Epidemiol Infect. 2008;136:1463-71.

29. Christen A, Navarro CM, Mausezahi D. Safe drinking water and clean air: An experimental study evaluating the concept of combining household water treatment and indoor air improvement using the Water Disinfection Stove (WADIS). Int J Hyg Environ Health. 2009;212(5):562-8.

30. Rosa G, Majorin F, Boisson S, Barstow C, Johnson M, Kirby M, Ngabo F, Thomas E, Clasen T. Assessing the Impact of Water Filters and Improved Cook Stoves on Drinking Water Quality and Household Air Pollution: A Randomised Controlled Trial in Rwanda. PLoS One. 2014;9(3):e91011.

31. Graf J, Togouet SZ, Kemka N, Niyitegeka D, Meierhofer R, Pieboji JG. Health gains from solar water disinfection (SODIS): evaluation of a water quality intervention in Yaoundé, Cameroon. J Water Health. 2010;8(4):779-96.

32. Boisson S, Kiyombo M, Sthreshley L, Tumba S, Makambo J, Clasen T. Field Assessment of a Novel Household-Based Water Filtration Device: A Randomised, Placebo-Controlled Trial in the Democratic Republic of Congo. PLoS One. 2010;5(9):e12613.

33. English R, Badcock JC, Giay T, Ngu T, Waters AM, Bennett SA. Effect of nutrition improvement project on morbidity from infectious diseases in preschool children in Vietnam: comparison with control commune. BMJ. 1997;315:1122

34. Faber M, Phungula MAS, Venter SL, Dhansay MA, Benade AJ S. Home gardens focusing on the production of yellow and dark-green leafy vegetables increase the serum retinol concentrations of 2-5-y-old children in South Africa. Am Soc Clin Nutr. 2002;76(5):1048-54.

35. Fenn B, Bulti AT, nduna T, Duffield A and Watson F. An evaluation of an operations research project to reduce childhood stunting in a food-insecure area in Ethiopia. 2012. 15;9: p. 1746-1754.

36. Creed-Kanashiro HM, Bartoini RM, Fukumoto MN, Uribe TG, Robert RC, Bentley ME. Formative research to develop a nutrition education intervention to improve dietary iron intake among women and adolescent girls through community kitchens in Lima Peru. J Nutr. 2003;133(11):3987S-91.

37. Pant CR, Pokharel GP, Curtale F, Pokhrel RP, Grosse RN, Lepkowski J, Muhilal, Bannister M, Gorstein J, Pak-Gorstein S, Atmarita, Tilden RL. Impact of nutrition education and mega-dose vitamin A supplementation on the health of children in Nepal. Bull World Health Org. 1996;74(5):533-45.

38. Santos I, Victora CG, Martines J, Gonçalves H, Gigante DP, Valle NJ, Pelto G. Nutrition counseling increases weight gain among Brazilian children. J Nutr. 2001;131:2866-73.

39. Moore JB, Pawloski L, Rodriguez C, Lumbi L, Ailinger R. The Effect of a Nutrition Education Program on the Nutritional Knowledge, Hemoglobin Levels, and Nutritional Status of Nicaraguan Adolescent Girls. Public Health Nurs. 2009;26(2):144-52.
40. Dohoo C, Guernsey J.R, Critchley K, and VanLeeuwen J. Pilot study on the impact of biogas as a fuel source on respiratory health of women on rural Kenyan smallholder dairy farms. J Environ Public Health. 2012.

41. Garfi M, Ferrer-Marti L, Perez I, Flotats X, Ferrer I. Codigestion of cow and guinea pig manure in low-cost tubular digesters at high altitude. Ecol Eng. 2011;37:2066-70.

42. Zhou Y, Zou Y, Li X, Chen S, Zhao Z, He F, et al. Lung function and incidence of chronic obstructive pulmonary disease after improved cooking fuels and kitchen ventilation: A 9 year prospective cohort study. PLoS Med. 2014;11(3):1-11.

43. Strunz EC, Addiss DG, Stocks ME, Ogden S, Utzinger J, Fewwman MC. Water, Sanitation, Hygiene, and Soil-Transmitted Helminth Infection: A Systematic Review and Meta-Analysis. PLoS Med. 2014;11(3):e1001620.

\section{Submit your next manuscript to BioMed Central and we will help you at every step:}

- We accept pre-submission inquiries

- Our selector tool helps you to find the most relevant journal

- We provide round the clock customer support

- Convenient online submission

- Thorough peer review

- Inclusion in PubMed and all major indexing services

- Maximum visibility for your research

Submit your manuscript at www.biomedcentral.com/submit
C Biomed Central 